\section{Validade e reprodutibilidade dos instrumentos de medida da atividade física do tipo self-report em adolescentes: uma revisão sistemática}

\author{
Validity and reliability of self-report instruments \\ for measuring physical activity in adolescents: \\ a systematic review
}

\author{
${ }_{1}$ Departamento de Educação \\ Física, Universidade Federal \\ da Paraíba, João Pessoa, \\ Brasil. \\ 2 Centro de Desportos, \\ Universidade Federal \\ de Santa Catarina, \\ Florianópolis, Brasil. \\ 3 Escola de Artes, Ciências e \\ Humanidades, Universidade \\ de São Paulo, São Paulo, \\ Brasil. \\ 4 Programa de Pós-graduação \\ em Educação Física \\ Universidade Federal de \\ Pelotas, Pelotas, Brasil. \\ 5 Programa de Pós-graduação \\ em Epidemiologia, \\ Universidade Federal de \\ Pelotas, Pelotas, Brasil. \\ Correspondência \\ J. C. Farias Júnior \\ Departamento de Educação \\ Física, Universidade Federal \\ da Paraíba. \\ Campus I, João Pessoa, PB \\ 88010-970, Brasil. \\ jcazuzajr@hotmail.com
}

\begin{abstract}
This was a systematic review of studies on the reliability and validity of self-report instruments for measuring physical activity, or subjective measurements, in adolescents (10-18 years). Searches were conducted in databases (MEDLINE, PsycInfo, SportsDiscus, Scopus, Web of Science, SciELO, Lilacs) and in the references of the retrieved articles. Sixty-six studies met the inclusion criteria. The majority were from North America, with only 5 from Brazil. Fifty-two different instruments were identified: 42 questionnaires, 6 diaries or logs, and 4 interviews. "Test-retest" reliability varied from 0.20 to 0.98; the majority (28/50) of the coefficients showed values $<0.70$. Validity coefficients showed wide variation (-0.13 to 0.88), with the majority $(64 / 84) \leq 0.50$. Only 3 instruments displayed correlations $\geq 0.70$. Various instruments were tested in adolescents, especially questionnaires. These instruments generally showed better "test-retest" reliability than validity.
\end{abstract}

Motor Activity; Adolescent; Reproducibility of Results; Validity of Tests; Methods
José Cazuza de Farias Júnior 1

Adair da Silva Lopes 2

Alex Antonio Florindo 3

Pedro C. Hallal 4,5

\section{Introdução}

Há um crescente reconhecimento da importância da avaliação do nível de prática de atividade física em estudos epidemiológicos 1,2. Entretanto, obter dados precisos sobre o nível de atividade física, sobretudo em crianças e adolescentes, é um grande desafio em razão da complexidade que caracteriza tal comportamento nesse grupo populacional $3,4,5$.

A atividade física representa qualquer movimento corporal produzido pelos músculos esqueléticos que resulta em gasto energético acima dos níveis de repouso (lazer, trabalho, deslocamentos, atividades do lar) 6,7. Esse conceito tem sido unanimemente aceito e amplamente adotado por pesquisadores de diversas áreas, em particular da área da saúde. O exercício físico é um tipo de atividade física, caracterizada por ser planejada, estruturada e repetitiva, que objetiva melhorar e/ou manter um ou mais componentes da aptidão física 7 .

Em estudos com o objetivo de estimar a prevalência de atividade física, identificar fatores associados, analisar a sua relação com diferentes desfechos de interesse e avaliar a efetividade de programas de intervenção é essencial dispor de instrumentos de medida da atividade física que apresentem propriedades psicométricas adequadas (reprodutibilidade e validade) ${ }^{4,5,8}$.

Há uma grande diversidade de métodos e técnicas para mensurar a atividade física, sen- 
do eles classificados como objetivos e subjetivos $4,5,8$. Os métodos objetivos (sensores de movimento, monitores de frequência cardíaca, água duplamente marcada - DLW, observação direta do comportamento), embora apresentem níveis considerados satisfatórios de reprodutibilidade $\mathrm{e}$ validade, possuem limitações em termos de logística e custo, podem ser reativos e mensuram apenas atividades físicas "atuais" ou "recentes" $8,9,10,11$.

Apesar do crescimento na utilização de acelerômetros e pedômetros para mensurar a atividade física em adolescentes 10,12, em estudos epidemiológicos a medida de atividade física tem sido efetuada, essencialmente, por métodos subjetivos (diários, questionários, entrevistas estruturadas), em particular em países de renda baixa e média 1,2 . Isso se deve à praticidade dessas medidas, ao baixo custo, por não serem reativos e permitirem, com algumas variações, mensurar as atividades físicas praticadas em um ou mais domínios, por diferentes períodos de tempo 4,5,8,11.

Vários estudos de revisão sobre métodos de medida da atividade física aplicados em adolescentes foram publicados $4,5,8,10,12,13$. Os que analisaram a reprodutibilidade e validade dos instrumentos do tipo self-report (medidas subjetivas da atividade física) não recorreram ao método de revisão sistemática $8,9,12$. Os estudos que utilizaram esse método foram realizados há quase uma década 8 ou mais 14 , ou analisaram as publicações durante um período curto de tempo (estudos publicados durante a década de 1990) 15.

Com intuito de preencher essa lacuna de conhecimento foram analisados, mediante método de revisão sistemática, os estudos de reprodutibilidade e validade dos instrumentos de medida da atividade física do tipo self-report testados em adolescentes (10-19 anos).

\section{Métodos}

As buscas foram realizadas em bases eletrônicas de dados, considerando o período de janeiro de 1980 a dezembro de 2007. A Tabela 1 apresenta as informações sobre as bases de dados consultadas, descritores e operadores utilizados na busca. O processo de revisão dos estudos está descrito na Figura 1.

Os seguintes critérios de inclusão foram adotados para esta revisão: (1) ser estudo de reprodutibilidade e/ou validade de instrumentos de medida da atividade física do tipo self-report; (2) incluir adolescentes de 10-19 anos ou média de idade dentro desse intervalo; (3) ter utilizado como critério de referência - "padrão-ouro" - ao menos uma medida objetiva e/ou subjetiva de atividade física e/ou de aptidão cardiorrespiratória; (4) ser artigo original de pesquisa desenvolvida com seres humanos, publicado em periódico indexado nas bases utilizadas, em inglês/ português/espanhol, durante janeiro de 1980 a dezembro de 2007.

Artigos de revisão, monografias, dissertações, teses, resumos, capítulos ou livros e ponto de vista/opinião de especialistas não foram incluídos. Também foram excluídos os estudos com adolescentes de grupos especiais e aqueles cujas medidas de reprodutibilidade e/ou validade foram apresentadas apenas como parte dos métodos (outros objetivos).

Estudos cujos resumos atenderam aos critérios de inclusão ou não apresentaram informações suficientes para que fossem excluídos de

Tabela 1

Bases de dados eletrônicas, descritores e operadores utilizados nas buscas.

\begin{tabular}{|c|c|c|}
\hline Base de dados & Descritores * & Operadores ** \\
\hline MEDLINE & 1. Instrumento: self-report, checklist, recall, $24 \mathrm{~h}$, interviews, & And, Or, Not \\
\hline Psyclnfo & questionnaire, diary, assessment, survey, measurement, log. & \\
\hline SportsDiscus & 2. Medida psicométrica: reproducibility, test-retest, reliability, validity, & \\
\hline Lilacs & validation, psychometric analysis, development. & \\
\hline Scopus & 3. Atividade física: physical activity, leisure activities, exercise, motor & \\
\hline Web of Science & activity, sports, transportation, working, organized, unorganized, daily & \\
\hline SciELO & physical activity, moderate, vigorous, energy expenditure. & \\
\hline & $\begin{array}{l}\text { 4. Grupo populacional: children, child, adolescent, adolescence, youth, } \\
\text { teen, teenager, high school, students, young. }\end{array}$ & \\
\hline
\end{tabular}

* Descritores em inglês/português, no título e/ou no resumo do artigo;

** Combinar os descritores e termos utilizados. 
Figura 1

Etapas do processo de revisão sistemática

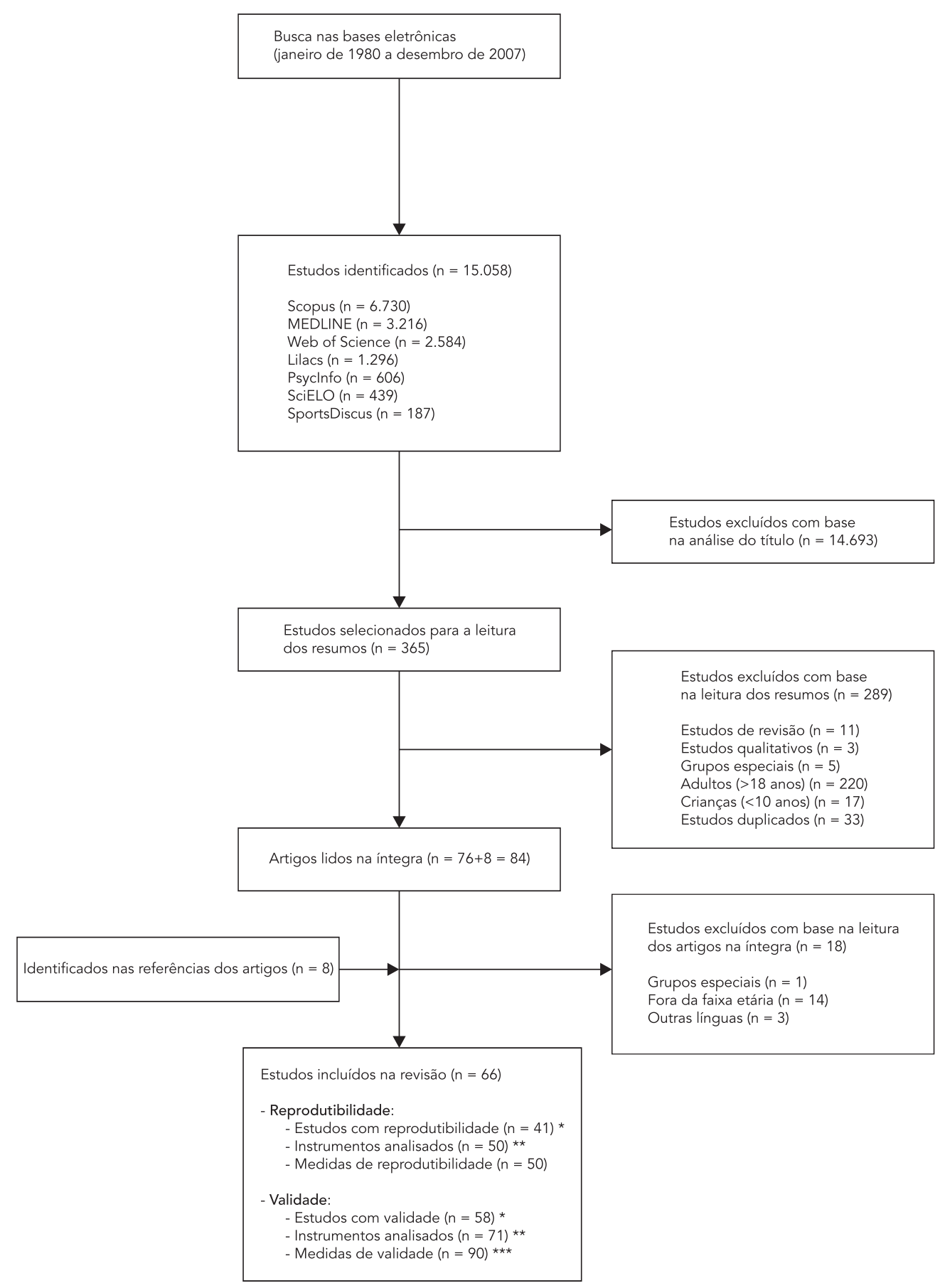

* Nem todos os estudos analisaram a reprodutibilidade e validade, simultaneamente;

** Alguns estudos analisaram mais de um instrumento (reprodutibilidade $n=6$; validade $n=10$ );

$\star \star \star$ Alguns instrumentos foram comparados contra mais de um critério de referência - "padrão-ouro" ( $n=15)$. 
forma imediata foram obtidos e lidos na íntegra. Efetuou-se uma revisão nas referências desses artigos com intuito de identificar algum estudo potencialmente relevante que não havia sido identificado na busca eletrônica.

A extração das informações nos artigos selecionados foi efetuada, em duplicata, por um único revisor, que utilizou uma ficha padronizada. As divergências observadas entre as duas revisões foram analisadas por mais dois pesquisadores, de forma independente, e foram resolvidas por meio de consenso. Informações que foram coletadas: (1) o primeiro autor do estudo, ano de publicação, número de sujeitos na amostra, total e por sexo, faixa etária, nome do país onde foi realizado o estudo, procedimento de seleção da amostra; (2) tipo de instrumento (diário, questionário, entrevista estruturada), nome original do instrumento, período de referência da sua medida (exemplo, um dia, semana típica, últimos sete dias, último ano), capacidade do instrumento de realizar estimativas do nível de atividade física de acordo com as recomendações atuais para adolescentes; (3) o critério de referência "padrão-ouro" - adotado na validação, a análise utilizada para estimar a reprodutibilidade "testereteste" e a validade, o intervalo entre as réplicas de aplicação do instrumento - "teste-reteste"; (4) a medida de reprodutibilidade e/ou validade, se o estudo comparou as medidas por sexo e idade dos adolescentes, e intensidade das atividades físicas (moderadas $v s$. vigorosas).

\section{Resultados}

Foram identificados 15.066 estudos, considerando os dois métodos de busca (bases eletrônicas $n=15.058$, referências dos artigos $n=8$ ). Sessenta e seis estudos atenderam aos critérios de inclusão. Dentre eles, 41 haviam analisado a reprodutibilidade "teste-reteste" e 58 a validade. Alguns estudos analisaram mais de um instrumento ( $\mathrm{n}=10$ ) 16,17,18,19,20,21,22,23,24,25 e, nos estudos com análise de validade, 15 instrumentos foram comparados contra mais de um critério de referência - "padrão-ouro" 16,23,26,27,28,29,30,31,32,33,34,35. Em função disso, nesta revisão, o "instrumento" será a unidade de análise e, por conseguinte, em algumas vezes os valores de "n" (ou o número de referências citadas) serão diferentes do número de estudos revisados (Figura 1).

A Tabela 2 apresenta uma descrição dos 66 estudos revisados. Foram analisados 79 instrumentos, sendo 16 diários, 57 questionários e 6 entrevistas estruturadas. A análise da reprodutibilidade foi efetuada em 50 instrumentos e da validade em 71 . Ou seja, em alguns instrumentos foi analisada apenas a reprodutibilidade $(\mathrm{n}=8)$ $36,37,38,39,40,41,42,43$, em outros a validade $(n=29)$ $16,18,21,25,28,30,31,32,33,44,45,46,47,48,49,50,51,52,53,54,55,56$, $57,58,59,60$.

Identificaram-se 52 instrumentos diferentes (42 questionários, 6 diários e 4 entrevistas estruturadas), mas apenas 11 haviam sido testados mais de uma vez $16,18,20,21,22,24,25,26,29,30,31,33,35,36,37$, $39,41,44,46,49,52,54,55,58,59,60,61,62,63,64,65,66,67$. A maioria destes instrumentos (33/52) 19,20,21,22,23,24,25, 27,28,32,35,38,40,41,46,47,50,51,53,55,56,57,61,64,68,69,70,71,72, 73,74 não permitia realizar estimativas do nível de atividade física dos adolescentes de acordo com as recomendações dos $300 \mathrm{~min} /$ semana 75 . Dos que realizavam tais estimativas, tirando uma entrevista (7-Day Physical Activity Recall) 16,24,60, todos eram questionários $(\mathrm{n}=18)$ 17,20,29,34,42,43, $45,48,61,67,76,77,78,79,80,81,82$.

O período de referência da medida de atividade física variou de um dia 22,23,25,26,27,30,32,35,38, $44,47,56,58,59,70,72$ aos últimos 12 meses 69 ou último ano 17,42,57,73,76,81. Os diários e as entrevistas mensuravam apenas as atividades físicas "atuais" ou "recentes”. Os questionários mensuravam tanto as atividades físicas "atuais" ou "recentes" ( $\mathrm{n}=25)$ $19,21,22,23,34,48,50,55,61,67,68,70,77,78,79$ quanto as atividades "habituais" ( $\mathrm{n}=17$ ) 17,20,24,28,42,53,57,69,71, $73,76,80,81,82$. Uma semana típica/habitual $(\mathrm{n}=9)$ $17,20,24,53,71,80,82$ e os últimos sete dias ou última semana $(\mathrm{n}=13$ ) 21,34,43,45,48,50,55,61,67,78,79 foram os períodos mais frequentemente utilizados pelos questionários.

\section{$\underline{\text { Reprodutibilidade }}$}

Foram identificados 41 estudos que haviam analisado a reprodutibilidade "teste-reteste" em 50 instrumentos. Trinta e dois instrumentos $17,18,22$, $23,26,29,34,37,38,40,43,61,63,64,65,66,67,69,71,72,73,74,77,78,79$, $80,81,82$ faziam parte de estudos publicados entre 2000 e 2007. A maioria foi testada em adolescentes de países da América do Norte (31/50) 18, $19,20,22,24,26,27,34,35,36,37,39,41,42,43,62,63,66,67,68,70,73,74$, 76,79 , sobretudo dos Estados Unidos $(\mathrm{n}=23) 18,19$, $22,24,26,27,34,35,36,37,41,42,43,63,66,67,68,70,73,76$, de ambos os sexos (44/50) 17,18,19,22,23,24,27,29,34,35,36,37,38, $39,40,41,42,43,61,62,63,64,65,66,67,69,70,71,72,73,74,76,77,78,79$, 80,81,82 e de diferentes faixas etárias (10-14 e 15-19 anos; 26/50) 17,19,20,24,27,35,36,37,42,62,64,65,69,73,77,78, $79,80,81,82$. O número de sujeitos na amostra variou de 1523 a 4.619 37; a maioria tinha 50 sujeitos ou mais (34/50) 17,18,20,22,24,29,34,35,36,37,38,39,41,43,62,64, $66,67,69,71,73,74,76,77,78,79,81,82$ e se tratava de amostras selecionadas por conveniência (25/50) 17,18,22,23, $24,26,27,34,36,37,40,43,62,64,66,68,69,70,71,72,73,77,79$ ou por participação voluntária (8/50) 19,38,41,42,63,67,80

Todas as medidas de reprodutibilidade foram estimadas mediante o procedimento "teste- 
Tabela 2

Síntese dos resultados dos estudos de reprodutibilidade e validade dos instrumentos de medida da atividade física do tipo self-report em adolescentes (10-19 anos).

\begin{tabular}{|c|c|c|c|c|c|c|c|}
\hline \multirow[t]{2}{*}{ Referências } & \multirow{2}{*}{$\begin{array}{l}\text { Tipo e nome } \\
\text { original do } \\
\text { instrumento }\end{array}$} & \multicolumn{2}{|c|}{ Reprodutibilidade "teste-reteste" } & \multicolumn{2}{|c|}{ Validade } & \multirow[t]{2}{*}{ Período * } & \multirow[t]{2}{*}{ Capacidade ** } \\
\hline & & $\begin{array}{c}\text { Características } \\
\text { da amostra }\end{array}$ & $\begin{array}{l}\text { Métodos e } \\
\text { resultados }\end{array}$ & $\begin{array}{c}\text { Características } \\
\text { da amostra }\end{array}$ & $\begin{array}{c}\text { Critério de referência } \\
\text { e resultados }\end{array}$ & & \\
\hline \multicolumn{8}{|c|}{ Diários/Logs } \\
\hline Lee \& Trost 64 & 3DPAR & $\begin{array}{c}\mathrm{N}=108(\mathrm{M} / \mathrm{F}), \\
\text { Cingapura } \\
\text { Idade: } 13-16 \\
\text { anos }\end{array}$ & $\begin{array}{c}\text { Teste-reteste, } \\
\text { 6-8h } \\
\mathrm{CCl}=0,88\end{array}$ & $\begin{array}{c}\mathrm{N}=221 \\
\text { (M: 105/F: 116), } \\
\text { Cingapura } \\
\text { Idade: 13-16 } \\
\text { anos }\end{array}$ & $\begin{array}{c}\text { Pedômetro, } 4 \mathrm{D} \\
\text { rho }=0,40\end{array}$ & U3D & 2 \\
\hline McMurray et al. 22 & 3DPAR & $\begin{array}{c}\mathrm{N}=320 \\
\text { (M: 114/F: 206), } \\
\text { Estados Unidos } \\
\text { Idade (média): } \\
\text { 12,5 anos; } \\
\text { DP }=1,1\end{array}$ & $\begin{array}{c}\text { Teste-reteste, 1D } \\
r=0,68\end{array}$ & $\begin{array}{c}\mathrm{N}=320 \\
\text { (M: 114/F: 206), } \\
\text { Estados Unidos } \\
\text { Idade (média): } \\
\text { 12,5 anos; } \\
\text { DP }=1,1\end{array}$ & $\begin{array}{c}\text { Acelerômetro, 4D } \\
r=0,30\end{array}$ & U3D & 2 \\
\hline Bouchard et al. 62 & 3DAR & $\begin{array}{c}\text { N = } 61(\mathrm{M} / \mathrm{F}) \\
\text { Canadá } \\
\text { Idade (média): } \\
14,6 \text { anos; } \\
\text { DP }=2,9\end{array}$ & $\begin{array}{c}\text { Teste-reteste, } \\
\text { 6-10D } \\
\mathrm{CCl}=0,91\end{array}$ & $\begin{array}{c}\mathrm{N}=150(\mathrm{M} / \mathrm{F}) \\
\text { Canadá } \\
\text { Idade (média): } \\
\begin{array}{c}14,6 \text { anos; } \mathrm{DP} \\
=2,9\end{array}\end{array}$ & $\begin{array}{c}\text { Cicloergômetro }{ }_{\text {PWC-150 }} \\
r=0,27\end{array}$ & $3 \mathrm{D}$ & 2 \\
\hline Argiropoulou et al. 61 & 3DAR & $\begin{array}{c}\mathrm{N}=40 \\
\text { (M: 23/F: 17), } \\
\text { Grécia } \\
\text { Idade: } 13-14 \\
\text { anos }\end{array}$ & $\begin{array}{c}\text { Teste-reteste, } 2 \mathrm{~S} \\
\mathrm{CCl}=0,97\end{array}$ & $\begin{array}{c}\mathrm{N}=40 \\
\text { (M: 23/F: 17), } \\
\text { Grécia } \\
\text { Idade: } 13-14 \\
\text { anos }\end{array}$ & $\begin{array}{l}\text { Acelerômetro, 7D } \\
\qquad r=0,63\end{array}$ & $3 \mathrm{D}$ & 2 \\
\hline Weston et al. 35 & PDPAR & $\begin{array}{c}\mathrm{N}=90(\mathrm{M} / \mathrm{F}), \\
\text { Estados Unidos } \\
\text { Idade: } 7-12 \\
\text { grade } * \star \star\end{array}$ & $\begin{array}{l}\text { Teste-reteste, } 1 \mathrm{~h} \\
\qquad r=0,98\end{array}$ & $\begin{array}{c}\mathrm{N}=48(\mathrm{M} / \mathrm{F}), \\
\text { Estados Unidos } \\
\text { Idade: } 7-12 \\
\text { grade } \\
\star \star \star\end{array}$ & $\begin{array}{l}\text { Pedômetro, 1D } \\
\qquad \begin{array}{c}r=0,88 \\
\text { Acelerômetro, 1D } \\
r=0,77\end{array}\end{array}$ & UD & 2 \\
\hline & & & & & $\begin{array}{l}\text { Monitoramento da } \\
\text { frequência cardíaca, } \\
\text { 1D } \\
r=0,53\end{array}$ & & \\
\hline Allor \& Pivarnik 26 & PDPAR & $\begin{array}{c}\mathrm{N}=46(\mathrm{~F}), \\
\text { Estados Unidos } \\
\text { Idade: } 12 \text { anos; } \\
\mathrm{DP}=0,6\end{array}$ & $\begin{array}{c}\text { Teste-reteste, 2D } \\
\mathrm{CCl}=0,98\end{array}$ & $\begin{array}{l}\mathrm{N}=46(\mathrm{~F}), \\
\text { Estados Unidos } \\
\text { Idade: } 12 \text { anos; } \\
\quad \mathrm{DP}=0,6\end{array}$ & $\begin{array}{c}\text { Acelerômetro, 2D } \\
\qquad \begin{array}{c}r=0,76 \\
\text { Monitoramento da } \\
\text { frequência cardíaca, } \\
2 \mathrm{D} \\
r=0,50\end{array}\end{array}$ & UD & 2 \\
\hline Welky et al. 25 & $\begin{array}{l}\text { ACTIVITYGRAM } \\
\text { Physical Activity }\end{array}$ & & & $\begin{array}{c}\mathrm{N}=28(\mathrm{M} / \mathrm{F}) \\
\text { Estados Unidos }\end{array}$ & $\begin{array}{c}\text { Acelerômetro, 3D } \\
r=0,50\end{array}$ & UD & 2 \\
\hline & Self-Report & & & $\begin{array}{l}\text { Idade (média): } \\
\begin{array}{c}12,4 \text { anos; } \\
\text { DP }=0,5\end{array}\end{array}$ & & & \\
\hline Pate et al. 54 & 3DPAR & & & $\begin{array}{c}\mathrm{N}=70(\mathrm{~F}), \\
\text { Estados Unidos } \\
\text { Idade: } 13-16 \\
\text { anos }\end{array}$ & $\begin{array}{l}\text { Acelerômetro, 7D } \\
\qquad r=0,49\end{array}$ & $3 U D$ & 2 \\
\hline
\end{tabular}

(continua) 
Tabela 2 (continuação)

\begin{tabular}{|c|c|c|c|c|c|c|c|}
\hline \multirow[t]{2}{*}{ Referências } & \multirow{2}{*}{$\begin{array}{l}\text { Tipo e nome } \\
\text { original do } \\
\text { instrumento }\end{array}$} & \multicolumn{2}{|c|}{ Reprodutibilidade "teste-reteste" } & \multicolumn{2}{|c|}{ Validade } & \multirow[t]{2}{*}{ Período * } & \multirow[t]{2}{*}{ Capacidade ** } \\
\hline & & $\begin{array}{c}\text { Características } \\
\text { da amostra }\end{array}$ & $\begin{array}{l}\text { Métodos e } \\
\text { resultados }\end{array}$ & $\begin{array}{l}\text { Características } \\
\text { da amostra }\end{array}$ & $\begin{array}{l}\text { Critério de referência } \\
\text { e resultados }\end{array}$ & & \\
\hline Bratteby et al. 46 & 3DAR & & & $\begin{array}{c}N=50 \\
\text { (M: 25/F: 25), } \\
\text { Suécia } \\
\text { Idade: } 15 \text { anos }\end{array}$ & $\begin{array}{c}\text { DLW, 7D } \\
\text { não há diferença na } \\
\text { estimativa do gasto } \\
\text { energético total entre } \\
\text { a DLW é o diário, } \\
\text { p }>0,05 \\
\text { B-A, } \\
\begin{array}{c}2 \mathrm{DP}=-3,47 \text { a } 3,77 \\
\mathrm{MJ} / \mathrm{d}\end{array}\end{array}$ & $3 \mathrm{D}$ & 2 \\
\hline Ekelund et al. 49 & 3DAR & & & $\begin{array}{c}\mathrm{N}=30 \\
\text { (M: 16/F: 14), } \\
\text { Suécia } \\
\text { Idade: } \\
\text { 14-16 anos }\end{array}$ & $\begin{array}{c}\text { Monitoramento da } \\
\text { frequência cardíaca, } \\
\text { 3D não há diferenças } \\
\text { no gasto energético } \\
\text { total e no tempo } \\
\text { de atividade física } \\
\text { moderada/vigorosa } \\
\text { entre diário e o } \\
\text { monitoramento da } \\
\text { frequência cardíaca, } \\
\text { p > 0,05 } \\
\text { B-A, } \\
\text { 2DP = -3,54 a } 2,74 \\
\text { MJ/d }\end{array}$ & $3 \mathrm{D}$ & 2 \\
\hline Anderson et al. 44 & PDPAR & & & $\begin{array}{c}\mathrm{N}=80 \\
\text { (M: 37/F: 43), } \\
\text { Estados Unidos } \\
\text { Idade (média): } \\
13,4 \text { anos; } \\
\text { DP }=0,5\end{array}$ & $\begin{array}{c}\text { Acelerômetro, 4D } \\
\text { rho }=0,42 \text { * }\end{array}$ & UD & 2 \\
\hline Trost et al. 59 & PDPAR & & & $\begin{array}{l}\mathrm{N}=37 \\
\text { (M: 18/F: 19), } \\
\text { Estados Unidos } \\
\text { Idade (média): } \\
\text { 10,8 anos; } \\
\text { DP }=0,1\end{array}$ & $\begin{array}{c}\text { Acelerômetro, 2D } \\
\text { rho }=0,41\end{array}$ & UD & 2 \\
\hline Trost et al. 58 & PDPAR & & & $\begin{array}{c}\mathrm{N}=122 \\
\text { (M: 57/F: 65), } \\
\text { Austrália } \\
\text { Idade (média): } \\
\text { 13,9 anos; } \\
\text { DP =1,2 }\end{array}$ & $\begin{array}{c}\text { Pedômetro, 1D } \\
\text { rho }=0,34\end{array}$ & UD & 2 \\
\hline Welky et al. 25 & PDPAR & & & $\begin{array}{l}\mathrm{N}=28(\mathrm{M} / \mathrm{F}) \\
\text { Estados Unidos } \\
\text { Idade (média): } \\
12,4 \text { anos; } \\
\mathrm{DP}=0,4\end{array}$ & $\begin{array}{l}\text { Acelerômetro, 3D } \\
\qquad r=0,65\end{array}$ & UD & 2 \\
\hline Rodriguez et al. 56 & $\begin{array}{l}\text { Self-Report } \\
\text { Activity Diary }\end{array}$ & & & $\begin{array}{c}\mathrm{n}=20 \text { (M: 13/F: 7), } \\
\text { França } \\
\text { Idade (média): } \\
11,2 \text { anos; } \\
\text { DP }=3,8\end{array}$ & $\begin{array}{l}\text { Monitoramento da } \\
\text { frequência cardíaca, } \\
\qquad \begin{array}{c}\text { 1D } \\
\text { rho }=0,73\end{array}\end{array}$ & UD & 2 \\
\hline
\end{tabular}

(continua) 
Tabela 2 (continuação)

\begin{tabular}{|c|c|c|c|c|c|c|c|}
\hline \multirow[t]{2}{*}{ Referências } & \multirow{2}{*}{$\begin{array}{l}\text { Tipo e nome } \\
\text { original do } \\
\text { instrumento }\end{array}$} & \multicolumn{2}{|c|}{ Reprodutibilidade "teste-reteste" } & \multicolumn{2}{|c|}{ Validade } & \multirow[t]{2}{*}{ Período * } & \multirow[t]{2}{*}{ Capacidade ** } \\
\hline & & $\begin{array}{l}\text { Características } \\
\text { da amostra }\end{array}$ & $\begin{array}{l}\text { Métodos e } \\
\text { resultados }\end{array}$ & $\begin{array}{l}\text { Características } \\
\text { da amostra }\end{array}$ & $\begin{array}{c}\text { Critério de referência } \\
\text { e resultados }\end{array}$ & & \\
\hline \multirow[t]{3}{*}{ Farias Junior et al. 40} & Adapted 3-Day & $N=45$ & Teste-reteste, 1D & & & $3 U D$ & 2 \\
\hline & Activity Record & $\begin{array}{l}\text { (M: 25/F: 20), } \\
\text { Brasil } \\
\text { Idade: 15-18 } \\
\text { anos }\end{array}$ & $\mathrm{CCl}=0,84$ & & & & \\
\hline & Questionários & & & & & & \\
\hline Sallis et al. 24 & GSPA & $\begin{array}{c}\mathrm{N}=102 \\
\text { (M: 50/F: 52), } \\
\text { Estados Unidos } \\
\text { Idade: } 10-16 \\
\text { anos }\end{array}$ & $\begin{array}{c}\text { Teste-reteste, } \\
2 \mathrm{~S} \\
\mathrm{CCl}=0,81\end{array}$ & $\begin{array}{l}\mathrm{N}=102 \\
\text { (M: } 50 / \mathrm{F}: 52), \\
\text { Estados Unidos } \\
\text { Idade: } 10-16 \\
\text { anos }\end{array}$ & $\begin{array}{c}\text { 7DPAR, 7D } \\
r=0,39\end{array}$ & ST & 2 \\
\hline Koo \& Rohan 20 & GSPA & $\begin{array}{c}\mathrm{N}=84(\mathrm{~F}), \\
\text { Canadá } \\
\text { Idade: } 7-15 \\
\text { anos }\end{array}$ & $\begin{array}{c}\text { Teste-reteste, } \\
11 \mathrm{M} \\
\text { rho }=0,48\end{array}$ & $\begin{array}{c}\mathrm{N}=479(\mathrm{~F}), \\
\text { Canadá } \\
\text { Idade: } 7-15 \\
\text { anos }\end{array}$ & $\begin{array}{l}\text { Questionário } \\
\text { rho }=0,33\end{array}$ & ST & 2 \\
\hline Gao et al. 18 & GSPA & $\begin{array}{c}\mathrm{N}=250 \\
\text { (M: 103/F: 147), } \\
\text { Estados Unidos } \\
\text { Idade: } 6-8 \\
\text { grade }{ }^{\star \star \star}\end{array}$ & $\begin{array}{c}\text { Teste-reteste, } \\
\text { 1S } \\
r=0,56\end{array}$ & $\begin{array}{c}\mathrm{N}=114 \\
\text { (M: 46/F: 68), } \\
\text { Estados Unidos } \\
\text { Idade: 6-8 } \\
\text { grade *** }\end{array}$ & $\begin{array}{c}\text { Acelerômetro, 7D } \\
\qquad r=0,19\end{array}$ & ST & 2 \\
\hline Eisenmann et al. 63 & GSPA & $\begin{array}{c}\mathrm{N}=31 \\
\text { (M: 17/F: 14), } \\
\text { Estados Unidos } \\
\text { Idade (média): } \\
\text { 10,6 anos; } \\
\text { DP = 0,2 }\end{array}$ & $\begin{array}{c}\text { Teste-reteste, } \\
\text { 1D } \\
r=0,62\end{array}$ & $\begin{array}{c}\mathrm{N}=31 \\
\text { (M: 17/F: 14), } \\
\text { Estados Unidos } \\
\text { Idade (média): } \\
\text { 10,6 anos; } \\
\text { DP = 0,2 }\end{array}$ & $\begin{array}{c}\text { Acelerômetro, 1D } \\
r=0,50\end{array}$ & ST & 2 \\
\hline Telford et al. 71 & CLASS & $\begin{array}{c}\mathrm{N}=111 \\
\text { (M: 41/F: 70), } \\
\text { Austrália } \\
\text { Idade: } 10-12 \\
\text { anos }\end{array}$ & $\begin{array}{c}\text { Teste-reteste, } \\
\text { 7D } \\
\mathrm{CCl}=0,30\end{array}$ & $\begin{array}{c}\mathrm{N}=111 \\
\text { (M: 41/F: 70), } \\
\text { Austrália } \\
\text { Idade: } 10-12 \\
\text { anos }\end{array}$ & $\begin{array}{c}\text { Acelerômetro, 8D } \\
\text { rho }=-0,04\end{array}$ & ST & 2 \\
\hline Hernández et al. 74 & CAINM & $\begin{array}{c}\mathrm{N}=114 \\
\text { (M: 47/F: 67), } \\
\text { México } \\
\text { Idade: } 10-14 \\
\text { anos }\end{array}$ & $\begin{array}{c}\text { Teste-reteste, } \\
6 \mathrm{M} \\
r=0,48\end{array}$ & $\begin{array}{c}\mathrm{N}=114 \\
(\mathrm{M}: 47 / \mathrm{F}: 67), \\
\text { México } \\
\text { Idade: } 10-14 \\
\text { anos }\end{array}$ & $\begin{array}{l}\text { Recordatório 24h, } \\
\qquad \begin{array}{c}\text { 7D } \\
r=0,02\end{array}\end{array}$ & UM & 2 \\
\hline Booth et al. 82 & APARQ & $\begin{array}{c}\mathrm{N}=226 \\
\text { (M: 138/F: 88), } \\
\text { Austrália } \\
\text { Idade: 13-15 } \\
\text { anos }\end{array}$ & $\begin{array}{l}\text { Teste-reteste, } \\
2 \mathrm{~S} \\
\mathrm{CCl}=0,69\end{array}$ & $\begin{array}{c}\mathrm{N}=2.026 \\
\text { (M: } 1.081 / \mathrm{F}: \\
945), \text { Austrália } \\
\text { Idade: } 13 \text { e } 15 \\
\text { anos }\end{array}$ & $\begin{array}{c}\text { Shuttle run test } \\
\text { (número de voltas) } \\
\text { rho }=0,22\end{array}$ & ST & 1 \\
\hline Ching \& Diez 68 & $\begin{array}{c}\text { Physical } \\
\text { Activity } \\
\text { Questionnaire }\end{array}$ & $\begin{array}{c}\mathrm{N}=40(\mathrm{~F}), \\
\text { Estados Unidos } \\
\text { Idade: } 8,5-12,7 \\
\text { anos }\end{array}$ & $\begin{array}{c}\text { Teste-reteste, } \\
12-16 \mathrm{D} \\
\mathrm{CCl}=0,51\end{array}$ & $\begin{array}{c}\mathrm{N}=40(\mathrm{~F}) \\
\text { Estados Unidos } \\
\text { Idade: } 8,5-12,7 \\
\text { anos }\end{array}$ & $\begin{array}{c}\text { Diário, 7D } \\
r=0,50\end{array}$ & $2 \mathrm{D}$ & 2 \\
\hline Aaron et al. 76 & $\begin{array}{l}\text { Questionnaire } \\
\text { to Assess Past } \\
\text { Year Physical } \\
\text { Activity in } \\
\text { Adolescent }\end{array}$ & $\begin{array}{c}\mathrm{N}=100 \\
\text { (M: 47/F: } 53), \\
\text { Estados Unidos } \\
\text { Idade: } 15-18 \\
\text { anos }\end{array}$ & $\begin{array}{c}\text { Teste-reteste, } \\
12 \mathrm{M} \\
\text { rho }=0,69\end{array}$ & $\begin{array}{c}\mathrm{N}=100 \\
\text { (M: 47/F: } 53), \\
\text { Estados Unidos } \\
\text { Idade: } 15-18 \\
\text { anos }\end{array}$ & $\begin{array}{c}\text { Teste da milha } \\
\text { (minutos) } \\
\text { rho }=0,32\end{array}$ & UA & 1 \\
\hline
\end{tabular}

(continua) 
Tabela 2 (continuação)

\begin{tabular}{|c|c|c|c|c|c|c|c|}
\hline \multirow[t]{2}{*}{ Referências } & \multirow{2}{*}{$\begin{array}{l}\text { Tipo e nome } \\
\text { original do } \\
\text { instrumento }\end{array}$} & \multicolumn{2}{|c|}{ Reprodutibilidade "teste-reteste" } & \multicolumn{2}{|c|}{ Validade } & \multirow[t]{2}{*}{ Período * } & \multirow[t]{2}{*}{ Capacidade ** } \\
\hline & & $\begin{array}{l}\text { Características } \\
\text { da amostra }\end{array}$ & $\begin{array}{l}\text { Métodos e } \\
\text { resultados }\end{array}$ & $\begin{array}{l}\text { Características } \\
\text { da amostra }\end{array}$ & $\begin{array}{l}\text { Critério de referência } \\
\text { e resultados }\end{array}$ & & \\
\hline Welky et al. 34 & YMCLS & $\begin{array}{c}\mathrm{N}=192 \\
\text { (M: 93/F: 99), } \\
\text { Estados Unidos } \\
\text { Idade: 9-13 anos }\end{array}$ & $\begin{array}{l}\text { Teste-reteste, } 1 \mathrm{~S} \\
\qquad \mathrm{CCl}=0,60\end{array}$ & $\begin{array}{c}\mathrm{N}=192 \\
\text { (M: 93/F: 99), } \\
\text { Estados Unidos } \\
\text { Idade: 9-13 anos }\end{array}$ & $\begin{array}{l}\text { Acelerômetro, 7D } \\
\qquad \begin{array}{c}r=0,24 \\
\text { Log de atividade } \\
\text { física, 7D } \\
r=0,46\end{array}\end{array}$ & U7D & 1 \\
\hline McMurray et al. 70 & $\begin{array}{l}\text { Computerized } \\
\text { Activity Recall }\end{array}$ & $\begin{array}{c}\mathrm{N}=22(\mathrm{M} / \mathrm{F}) \\
\text { Estados Unidos } \\
\text { Idade (média): } \\
11,8 \text { anos; } \\
\mathrm{DP}=1,0\end{array}$ & $\begin{array}{c}\text { Teste-reteste, } \\
1 \text {-2S } \\
\mathrm{CCl}=0,82\end{array}$ & $\begin{array}{l}\quad \mathrm{N}=45 \\
\text { (M: 20/F: 25), } \\
\text { Estados Unidos } \\
\text { Idade (média): } \\
\text { 11,8 anos; } \\
\text { DP }=1,0\end{array}$ & $\begin{array}{c}\text { Acelerômetro, 5D } \\
\qquad r=0,51\end{array}$ & UD & 2 \\
\hline Prochaska et al.66 & PACE+ & $\begin{array}{c}\mathrm{N}=138 \\
\text { (M: 48/F: 90), } \\
\text { Estados Unidos } \\
\text { Idade (média): } \\
\text { 12,1 anos; } \\
\text { DP }=0,9\end{array}$ & $\begin{array}{l}\text { Teste-reteste, } \\
<24 \mathrm{~h} \\
\mathrm{CCl}=0,77\end{array}$ & $\begin{array}{c}\mathrm{N}=138 \\
\text { (M: 48/F: 90), } \\
\text { Estados Unidos } \\
\text { Idade (média): } \\
\text { 12,1 anos; } \\
\text { DP }=0,9\end{array}$ & $\begin{array}{c}\text { Acelerômetro, 5D } \\
\qquad r=0,40\end{array}$ & $\mathrm{MC}$ & 1 \\
\hline Nahas et al. 29 & PACE+ & $\begin{array}{c}N=122 \\
\text { (M: 44/F: 78), } \\
\text { Brasil } \\
\text { Idade: } 15-24 \\
\text { anos }\end{array}$ & $\begin{array}{l}\text { Teste-reteste, } 1 \mathrm{~S} \\
\qquad \mathrm{CCl}=0,93\end{array}$ & $\begin{array}{c}\mathrm{N}=65 \\
\text { (M: 26/F: 39), } \\
\text { Brasil } \\
\text { Idade: } 15-24 \\
\text { anos }\end{array}$ & $\begin{array}{c}\text { Pedômetro, 7D } \\
\text { rho }=0,23 \\
\text { Recordatório, 7D } \\
\text { rho }=0,41\end{array}$ & MC & 1 \\
\hline Ridley et al.23 & CDPAQ & $\begin{array}{c}\mathrm{N}=15 \\
\text { (M: 7/F: 8), } \\
\text { Austrália } \\
\text { Idade (média): } \\
\text { 12,1 anos; } \\
\mathrm{DP}=0,5\end{array}$ & $\begin{array}{l}\text { Teste-reteste, } \\
<24 \mathrm{~h} \\
\mathrm{CCl}=0,94\end{array}$ & $\begin{array}{c}N=30 \\
\text { (M: 15/F: 15), } \\
\text { Austrália } \\
\text { Idade (média): } \\
\text { 11,96 anos; } \\
\text { DP }=0,5\end{array}$ & $\begin{array}{l}\text { Acelerômetro, 1D } \\
\qquad \begin{array}{c}r=0,45 \\
\text { Monitoramento da } \\
\text { frequência cardíaca, } \\
\text { 1D } \\
r=0,66\end{array}\end{array}$ & UD & 2 \\
\hline Argiropoulou et al. 61 & PALQ & $\begin{array}{c}\mathrm{N}=40 \\
\text { (M: 23/F: 17), } \\
\text { Grécia } \\
\text { Idade: } 13-14 \\
\text { anos }\end{array}$ & $\begin{array}{l}\text { Teste-reteste, } 2 \mathrm{~S} \\
\qquad \mathrm{CCl}=0,52\end{array}$ & $\begin{array}{c}\mathrm{N}=40 \\
\text { (M: 23/F: 17), } \\
\text { Grécia } \\
\text { Idade: } 13-14 \\
\text { anos }\end{array}$ & $\begin{array}{c}\text { Acelerômetro, 7D } \\
\qquad r=0,53\end{array}$ & U7D & 1 \\
\hline Philippaerts et al. 80 & $\begin{array}{c}\text { Flemish } \\
\text { Physical Activity } \\
\text { Questionnaire }\end{array}$ & $\begin{array}{c}\mathrm{N}=33 \\
\text { (M: 10/F: 23), } \\
\text { Bélgica } \\
\text { Idade: } 12-18 \\
\text { anos }\end{array}$ & $\begin{array}{l}\text { Teste-reteste, 9D } \\
\qquad \mathrm{CCl}=0,68\end{array}$ & $\begin{array}{c}\mathrm{N}=33 \\
\text { (M: 10/F: 23), } \\
\text { Bélgica } \\
\text { Idade: } 12-18 \\
\text { anos }\end{array}$ & $\begin{array}{c}\text { Acelerômetro, 7D } \\
\qquad r=0,45\end{array}$ & ST & 1 \\
\hline Barbosa et al. 81 & QAPACE & $\begin{array}{c}\mathrm{N}=121 \\
(\mathrm{M}: 56 / \mathrm{F}: \text { 65), } \\
\text { Colômbia } \\
\text { Idade: } 8-16 \text { anos }\end{array}$ & $\begin{array}{c}\text { Teste-reteste, } \\
90 \mathrm{D} \\
\mathrm{CCl}=0,96\end{array}$ & $\begin{array}{c}\mathrm{N}=36 \\
\text { (M: 18/F: 18), } \\
\text { Colômbia } \\
\text { Idade: } 8-16 \text { anos }\end{array}$ & $\begin{array}{l}\mathrm{VO}_{2} \max \\
r=0,63\end{array}$ & UA & 1 \\
\hline Wong et al. 79 & SHAPES & 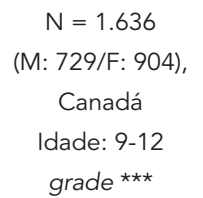 & $\begin{array}{c}\text { Teste-reteste, } 1 \mathrm{~S} \\
\text { kappa }=0,37\end{array}$ & $\begin{array}{c}\mathrm{N}=67 \\
\text { (M: 34/F: 33), } \\
\text { Canadá } \\
\text { Idade: } 9-12 \\
\text { grade }{ }^{\star \star \star}\end{array}$ & $\begin{array}{c}\text { Acelerômetro, 7D } \\
\text { rho }=0,44\end{array}$ & U7D & 1 \\
\hline
\end{tabular}

(continua) 
Tabela 2 (continuação)

\begin{tabular}{|c|c|c|c|c|c|c|c|}
\hline \multirow[t]{2}{*}{ Referências } & \multirow{2}{*}{$\begin{array}{l}\text { Tipo e nome } \\
\text { original do } \\
\text { instrumento }\end{array}$} & \multicolumn{2}{|c|}{ Reprodutibilidade "teste-reteste" } & \multicolumn{2}{|c|}{ Validade } & \multirow[t]{2}{*}{ Período * } & \multirow[t]{2}{*}{ Capacidade ** } \\
\hline & & $\begin{array}{l}\text { Características } \\
\text { da amostra }\end{array}$ & $\begin{array}{l}\text { Métodos e } \\
\text { resultados }\end{array}$ & $\begin{array}{l}\text { Características } \\
\text { da amostra }\end{array}$ & $\begin{array}{l}\text { Critério de referência } \\
\text { e resultados }\end{array}$ & & \\
\hline Guedes et al. 77 & IPAQ & $\begin{array}{c}\mathrm{N}=161 \\
\text { (M: 69/F: 92), } \\
\text { Brasil } \\
\text { Idade: } 12-18 \\
\text { anos }\end{array}$ & $\begin{array}{c}\text { Teste-reteste, } 2 \mathrm{~S} \\
\text { rho }=0,59\end{array}$ & $\begin{array}{c}\mathrm{N}=161 \\
\text { (M: 69/F: 92), } \\
\text { Brasil } \\
\text { Idade: } 12-18 \\
\text { anos }\end{array}$ & $\begin{array}{l}3 \mathrm{DAR}, 4 \mathrm{D} \\
\text { rho }=0,26\end{array}$ & US & 1 \\
\hline \multirow[t]{2}{*}{ Florindo et al. 17} & ST & $\begin{array}{c}\mathrm{N}=94 \\
\text { (M: 30/F: 64), } \\
\text { Brasil } \\
\text { Idade: } 11-16 \\
\text { anos }\end{array}$ & $\begin{array}{l}\text { Teste-reste, 15D } \\
\qquad \mathrm{CCl}=0,61\end{array}$ & $\begin{array}{c}\mathrm{N}=94 \\
\text { (M: 30/F: 64), } \\
\text { Brasil } \\
\text { Idade: } 11-16 \\
\text { anos }\end{array}$ & $\begin{array}{c}\text { Shuttle run test } \\
\text { rho }=0,15\end{array}$ & ST & 1 \\
\hline & UA & $\begin{array}{c}\mathrm{N}=94 \\
\text { (M: 30/F: 64), } \\
\text { Brasil } \\
\text { Idade: } 11-16 \\
\text { anos }\end{array}$ & $\begin{array}{l}\text { Teste-reste, 15D } \\
\mathrm{CCl}=0,68\end{array}$ & $\begin{array}{c}\mathrm{N}=94 \\
\text { (M: 30/F: 64), } \\
\text { Brasil } \\
\text { Idade: } 11-16 \\
\text { anos }\end{array}$ & $\begin{array}{c}\text { Shuttle run test } \\
\text { rho }=0,23\end{array}$ & UA & 1 \\
\hline Booth et al. 78 & WHO HBSC & $\begin{array}{c}N=226 \\
\text { (M: 138/F: } 88), \\
\text { Austrália } \\
\text { Idade: } 13,7-15,7 \\
\text { anos }\end{array}$ & $\begin{array}{c}\text { Teste-reteste, } 2 \mathrm{~S} \\
\text { kappa }=0,38\end{array}$ & $\begin{array}{c}\mathrm{N}=2.026 \\
\text { (M: 1.082/F: } 944), \\
\text { Austrália } \\
\text { Idade: } 13,7-15,7 \\
\text { anos }\end{array}$ & $\begin{array}{l}\text { Shuttle run test, maior } \\
\text { número de voltas } \\
\text { nos adolescentes } \\
\text { fisicamente mais } \\
\text { ativos }\end{array}$ & U7D & 1 \\
\hline Guedes et al. 69 & $\begin{array}{c}\text { Baecke } \\
\text { Questionnaire } \\
\text { of Habitual } \\
\text { Physical Activity }\end{array}$ & $\begin{array}{c}\mathrm{N}=161 \\
\text { (M: 69/F: 92), } \\
\text { Brasil } \\
\text { Idade: } 12-18 \\
\text { anos }\end{array}$ & $\begin{array}{c}\text { Teste-reteste, } 2 \mathrm{~S} \\
\mathrm{CCl}=0,73\end{array}$ & $\begin{array}{c}\mathrm{N}=161 \\
\text { (M: 69/F: 92), } \\
\text { Brasil } \\
\text { Idade: } 12-18 \\
\text { anos }\end{array}$ & $\begin{array}{c}\text { Diário, 4D } \\
k=0,44\end{array}$ & U12M & 2 \\
\hline \multirow[t]{3}{*}{ Koo \& Rohan 20} & PS & $\begin{array}{c}\mathrm{N}=84(\mathrm{~F}) \\
\text { Canadá } \\
\text { Idade: } 7-15 \text { anos }\end{array}$ & $\begin{array}{c}\text { Teste-reteste, } \\
11 \mathrm{M} \\
\text { rho }=0,44\end{array}$ & $\begin{array}{c}\text { N }=479(\mathrm{~F}) \\
\text { Canadá } \\
\text { Idade: } 7-15 \text { anos }\end{array}$ & $\begin{array}{l}\text { Questionário } \\
\text { rho = 0,29 }\end{array}$ & ST & 2 \\
\hline & SS & $\begin{array}{c}\mathrm{N}=84(\mathrm{~F}), \\
\text { Canadá } \\
\text { Idade: } 7-15 \text { anos }\end{array}$ & $\begin{array}{c}\text { Teste-reteste, } \\
11 \mathrm{M} \\
\text { rho }=0,59\end{array}$ & $\begin{array}{c}\text { N }=479(\mathrm{~F}) \\
\text { Canadá } \\
\text { Idade: } 7-15 \text { anos }\end{array}$ & $\begin{array}{l}\text { Questionário } \\
\text { rho }=0,16\end{array}$ & ST & 2 \\
\hline & SAS & $\begin{array}{c}\mathrm{N}=84(\mathrm{~F}) \\
\text { Canadá } \\
\text { Idade: } 7-15 \text { anos }\end{array}$ & $\begin{array}{c}\text { Teste-reteste, } \\
11 \mathrm{M} \\
\text { rho }=0,53\end{array}$ & $\begin{array}{c}\mathrm{N}=479(\mathrm{~F}) \\
\text { Canadá } \\
\text { Idade: } 7-15 \text { anos }\end{array}$ & $\begin{array}{l}\text { Questionário } \\
\text { rho }=0,26\end{array}$ & ST & 1 \\
\hline \multirow[t]{2}{*}{ Janz et al. 19} & $3 \mathrm{DSR}$ & $\begin{array}{c}\mathrm{N}=30 \\
\text { (M: 15/F: 15), } \\
\text { Estados Unidos } \\
\text { Idade: 7-15 anos }\end{array}$ & $\begin{array}{l}\text { Teste-reteste, } 1 \mathrm{M} \\
\qquad \mathrm{CCl}=0,30\end{array}$ & $\begin{array}{c}\mathrm{N}=30 \\
\text { (M: 15/F: 15), } \\
\text { Estados Unidos } \\
\text { Idade: 7-15 anos }\end{array}$ & $\begin{array}{c}\text { Acelerômetro, } 6 \mathrm{D} \\
\qquad r=0,48\end{array}$ & $3 \cup \mathrm{D}$ & 2 \\
\hline & 3DAR & $\begin{array}{c}\mathrm{N}=30 \\
\text { (M: 15/F: 15), } \\
\text { Estados Unidos } \\
\text { Idade: } 7-15 \text { anos }\end{array}$ & $\begin{array}{c}\text { Teste-reteste, } 1 \mathrm{M} \\
\qquad \mathrm{CCl}=0,54\end{array}$ & $\begin{array}{c}\mathrm{N}=30 \\
\text { (M: 15/F: 15), } \\
\text { Estados Unidos } \\
\text { Idade: 7-15 anos }\end{array}$ & $\begin{array}{c}\text { Acelerômetro, } 6 \mathrm{D} \\
\quad r=0,39\end{array}$ & $3 \cup \mathrm{U}$ & 2 \\
\hline Troped et al. 67 & $\begin{array}{c}\text { Youth Risk } \\
\text { Behavior Survey } \\
\text { Questionnaire } \\
\text { - } 2003\end{array}$ & $\begin{array}{l}\mathrm{N}=128(\mathrm{M} / \mathrm{F} \text { ), } \\
\text { Estados Unidos } \\
\text { Idade (média): } \\
\text { 12,6 anos; } \\
\text { DP =0,6 }\end{array}$ & $\begin{array}{l}\text { Teste-reste, } 1-2 \mathrm{~S} \\
\mathrm{CCl}=0,49\end{array}$ & $\begin{array}{c}\mathrm{N}=125 \\
\text { (M: 60/F: 65), } \\
\text { Estados Unidos } \\
\text { Idade (média): } \\
\text { 12,6 anos; } \\
\text { DP = 0,6 }\end{array}$ & $\begin{array}{c}\text { Acelerômetro, 7D } \\
S E=0,20 \text { e } E S=0,82\end{array}$ & U7D & 1 \\
\hline
\end{tabular}

(continua) 
Tabela 2 (continuação)

\begin{tabular}{|c|c|c|c|c|c|c|c|}
\hline \multirow[t]{2}{*}{ Referências } & \multirow{2}{*}{$\begin{array}{l}\text { Tipo e nome } \\
\text { original do } \\
\text { instrumento }\end{array}$} & \multicolumn{2}{|c|}{ Reprodutibilidade "teste-reteste" } & \multicolumn{2}{|c|}{ Validade } & \multirow[t]{2}{*}{ Período * } & \multirow[t]{2}{*}{ Capacidade ** } \\
\hline & & $\begin{array}{l}\text { Características } \\
\text { da amostra }\end{array}$ & $\begin{array}{l}\text { Métodos e } \\
\text { resultados }\end{array}$ & $\begin{array}{l}\text { Características } \\
\text { da amostra }\end{array}$ & $\begin{array}{l}\text { Critério de referência } \\
\text { e resultados }\end{array}$ & & \\
\hline Argiropoulou 61 & $\begin{array}{l}\text { Four by One- } \\
\text { Day Recall } \\
\text { Physical Activity } \\
\text { Questionnaire }\end{array}$ & $\begin{array}{c}\mathrm{N}=40 \\
\text { (M: 23/F: 17), } \\
\text { Grécia } \\
\text { Idade: } 13-14 \\
\text { anos }\end{array}$ & $\begin{array}{l}\text { Teste-reteste, } 2 \mathrm{~S} \\
\qquad \mathrm{CCl}=0,70\end{array}$ & $\begin{array}{c}N=40 \\
\text { (M: 23/F: 17), } \\
\text { Grécia } \\
\text { Idade: } 13-14 \\
\text { anos }\end{array}$ & $\begin{array}{c}\text { Acelerômetro, 7D } \\
\qquad r=0,62\end{array}$ & $3 U D$ & 2 \\
\hline Treuth 73 & $\begin{array}{l}\text { Fels Physical } \\
\text { Activity } \\
\text { Questionnaire }\end{array}$ & $\begin{array}{c}\mathrm{N}=229 \\
\text { (M: 99/F: 130), } \\
\text { Estados Unidos } \\
\text { Idade: 7-19 anos }\end{array}$ & $\begin{array}{l}\text { Teste-reteste, 6D } \\
\qquad \mathrm{CCl}=0,66\end{array}$ & $\begin{array}{c}\mathrm{N}=229 \\
\text { (M: 99/F: 130), } \\
\text { Estados Unidos } \\
\text { Idade: 7-19 anos }\end{array}$ & $\begin{array}{c}\text { Acelerômetro, 6D } \\
\text { rho }=0,21\end{array}$ & UA & 2 \\
\hline Ridley et al. 72 & MARCA & $\begin{array}{c}\mathrm{N}=32 \\
\text { (M: 18/F: 14), } \\
\text { Austrália } \\
\text { Idade: 9-13,5 } \\
\text { anos }\end{array}$ & $\begin{array}{l}\text { Teste-reteste, 1D } \\
\qquad \mathrm{CCl}=0,94\end{array}$ & $\begin{array}{c}\mathrm{N}=66 \\
\text { (M: 33/F: 33), } \\
\text { Austrália } \\
\text { Idade: } 9-15 \text { anos }\end{array}$ & $\begin{array}{c}\text { Acelerômetro, 1D } \\
\text { rho }=0,35\end{array}$ & UD & 2 \\
\hline Garcia et al. 27 & CAAL & $\begin{array}{c}\mathrm{N}=25(\mathrm{M} / \mathrm{F}) \\
\text { Estados Unidos } \\
\text { Idade: } 11-15 \\
\text { anos }\end{array}$ & $\begin{array}{l}\text { Teste-reteste, 1D } \\
\qquad \mathrm{CCl}=0,95\end{array}$ & $\begin{array}{c}\mathrm{N}=94(\mathrm{M} / \mathrm{F}) \\
\text { Estados Unidos } \\
\text { Idade: } 11-15 \\
\text { anos }\end{array}$ & $\begin{array}{l}\text { Acelerômetro, 5D } \\
\begin{array}{l}r=0,39 \\
\text { Step test } \\
r=0,14\end{array}\end{array}$ & UD & 2 \\
\hline McMurray et al. 22 & SAPAC & $\begin{array}{c}\mathrm{N}=320 \\
\text { (M: 206/F: 114), } \\
\text { Estados Unidos } \\
\text { Idade (média): } \\
\text { 12,5 anos; } \\
\text { DP }=1,1\end{array}$ & $\begin{array}{c}\text { Teste-reteste, 1D } \\
r=0,67\end{array}$ & $\begin{array}{c}\mathrm{N}=320 \\
\text { (M: 206/F: 114), } \\
\text { Estados Unidos } \\
\text { Idade (média): } \\
\text { 12,5 anos; } \\
\text { DP }=1,1\end{array}$ & $\begin{array}{l}\text { Acelerômetro, 4D } \\
\qquad r=0,24\end{array}$ & UD & 1 \\
\hline Mota et al. 65 & $\begin{array}{c}\text { Weekly activity } \\
\text { checklist }\end{array}$ & $\begin{array}{c}\mathrm{N}=30(\mathrm{M} / \mathrm{F}), \\
\text { Portugal } \\
\text { Idade: } 8-16 \text { anos }\end{array}$ & $\begin{array}{l}\text { Teste-reteste, } 1 \mathrm{~S} \\
\qquad \mathrm{CCl}=0,70\end{array}$ & $\begin{array}{c}\mathrm{N}=109 \\
(\mathrm{M}: 42 / \mathrm{F}: 67) \\
\text { Portugal } \\
\text { Idade: } 8-16 \text { anos }\end{array}$ & $\begin{array}{l}\text { Acelerômetro, 3D } \\
\qquad r=0,30\end{array}$ & US & 2 \\
\hline Scerpella et al. 31 & GSPA & & & $\begin{array}{c}\mathrm{N}=61(\mathrm{~F}), \\
\text { Estados Unidos } \\
\text { Idade (média): } \\
\text { 10,2 anos; } \\
\text { DP = 1,1 }\end{array}$ & $\begin{array}{c}\text { Checklist, 6D } \\
\text { rho }=0,25 \\
\text { Acelerômetro, 3D } \\
\text { rho }=0,10\end{array}$ & ST & 2 \\
\hline Ridder et al. 55 & $\begin{array}{c}\text { Weight Bearing } \\
\text { Activity } \\
\text { Questionnaire }\end{array}$ & & & $\begin{array}{c}\mathrm{N}=72 \\
\text { (M: 35/F: 37), } \\
\text { Holanda } \\
\text { Idade: } 8-14 \text { anos }\end{array}$ & $\begin{array}{c}\text { Acelerômetro, 4-5D } \\
\text { rho }=0,44\end{array}$ & U7D & 2 \\
\hline Verheul et al. 33 & $\begin{array}{l}\text { Weight Bearing } \\
\text { Activity } \\
\text { Questionnaire }\end{array}$ & & & $\begin{array}{c}\mathrm{N}=26(\mathrm{~F}) \\
\text { Holanda } \\
\text { Idade: } 11-15 \\
\text { anos }\end{array}$ & $\begin{array}{c}\text { Acelerômetro, 3D, } \\
\begin{array}{c}r=0,12 \\
\text { 3DAR, 3D } \\
r=0,57\end{array}\end{array}$ & U7D & 2 \\
\hline Ekelund et al. 48 & SAPAQ & & & $\begin{array}{c}\mathrm{n}=49 \\
\text { (M: 18/F: 31), } \\
\text { Suécia } \\
\text { Idade (média): } \\
\text { 16,8 anos; } \\
\text { DP }=0,4\end{array}$ & $\begin{array}{l}\text { Acelerômetro, 7D } \\
\qquad r=0,51\end{array}$ & U7D & 1 \\
\hline
\end{tabular}

(continua) 
Tabela 2 (continuação)

\begin{tabular}{|c|c|c|c|c|c|c|c|}
\hline \multirow[t]{2}{*}{ Referências } & \multirow{2}{*}{$\begin{array}{l}\text { Tipo e nome } \\
\text { original do } \\
\text { instrumento }\end{array}$} & \multicolumn{2}{|c|}{ Reprodutibilidade "teste-reteste" } & \multicolumn{2}{|c|}{ Validade } & \multirow[t]{2}{*}{ Período * } & \multirow[t]{2}{*}{ Capacidade ** } \\
\hline & & $\begin{array}{c}\text { Características } \\
\text { da amostra }\end{array}$ & $\begin{array}{l}\text { Métodos e } \\
\text { resultados }\end{array}$ & $\begin{array}{l}\text { Características } \\
\text { da amostra }\end{array}$ & $\begin{array}{l}\text { Critério de referência } \\
\text { e resultados }\end{array}$ & & \\
\hline \multirow[t]{6}{*}{ Kowalski 50} & PAQ-A & & & $N=85$ & Acelerômetro, 7D & U7D & 2 \\
\hline & & & & (M: 41/F: 44), & $r=0,33$ & & \\
\hline & & & & Canadá & 7DPAR & & \\
\hline & & & & Idade: $13-20$ & $r=0,51$ & & \\
\hline & & & & anos & GSPA, 7D & & \\
\hline & & & & & $r=0,57$ & & \\
\hline \multirow[t]{12}{*}{ Kowalski et al. 21} & PAQ-C & & & $N=97$ & 7-day recall, 7D & U7D & 2 \\
\hline & & & & (M: 41/F: 56), & $r=0,43$ & & \\
\hline & & & & Canadá & Acelerômetro, 7D & & \\
\hline & & & & Idade: 9-14 anos & $r=0,39$ & & \\
\hline & & & & & GSPA, 7D & & \\
\hline & & & & & $r=0,41$ & & \\
\hline & & & & & Step test & & \\
\hline & & & & & $r=0,28$ & & \\
\hline & PAQ-C & & & $N=89$ & Tempo de atividade & U7D & 2 \\
\hline & & & & (M: 38/F: 51), & física moderada/ & & \\
\hline & & & & Canadá & vigorosa, 7D & & \\
\hline & & & & Idade: 8-13 anos & $r=0,47$ & & \\
\hline \multirow[t]{4}{*}{ Moore et al. 52} & PAQ-C & & & $N=991$ & Step test & U7D & 2 \\
\hline & & & & (M: 494/F: 490), & $r=0,08$ & & \\
\hline & & & & Estados Unidos & & & \\
\hline & & & & Idade: 8-14 anos & & & \\
\hline \multirow[t]{6}{*}{ Crocker et al. 16} & PAQ-C & & & $N=61$ & Acelerômetro - R3D, & U7D & 2 \\
\hline & & & & (M: 27/F: 34), & $7 D$ & & \\
\hline & & & & Canadá & $r=0,13$ & & \\
\hline & & & & Idade (média): & 7DPAR, 7D & & \\
\hline & & & & 11,2 anos; & $r=0,39$ & & \\
\hline & & & & $\mathrm{DP}=0,6$ & & & \\
\hline \multirow[t]{5}{*}{ Gao et al. 18} & Youth Risk & & & $N=114$ & Acelerômetro, 7D & U7D & 1 \\
\hline & Behavior Survey & & & (M: 46/F: 68), & $r=0,10$ & & \\
\hline & Questionnaire & & & Estados Unidos & & & \\
\hline & -1999 & & & Idade: 6-8 & & & \\
\hline & & & & grade $^{\star \star \star}$ & & & \\
\hline \multirow[t]{5}{*}{ Slinde et al. 57} & Minnesota & & & $N=35$ & DLW, 14D & UA & 2 \\
\hline & Leisure Time & & & (M: 18/F: 17), & rho $=0,49$ & & \\
\hline & Physical Activity & & & Suécia & & & \\
\hline & Questionnaire & & & Idade: 15-17 & & & \\
\hline & & & & anos & & & \\
\hline \multirow[t]{4}{*}{ Arvidsson et al. 45} & Physical Activity & & & $N=33$ & DLW, 14D & U7D & 1 \\
\hline & Questionnaire & & & (M: 17/F: 16), & $r=0,62$ & & \\
\hline & for Adolescent & & & Suécia & & & \\
\hline & & & & Idade: 15 anos & & & \\
\hline \multirow[t]{6}{*}{ Sallis et al. 30} & SAPAC & & & $N=125$ & Monitoramento da & UD & 1 \\
\hline & & & & (M: 55/F: 70), & frequência cardíaca, & & \\
\hline & & & & Estados Unidos & 1D & & \\
\hline & & & & Idade (média): & $r=0,31$ & & \\
\hline & & & & 10,9 anos; & Acelerômetro, 1D & & \\
\hline & & & & $\mathrm{DP}=0,5$ & $r=0,54$ & & \\
\hline
\end{tabular}

(continua) 
Tabela 2 (continuação)

\begin{tabular}{|c|c|c|c|c|c|c|c|}
\hline \multirow[t]{2}{*}{ Referências } & \multirow{2}{*}{$\begin{array}{l}\text { Tipo e nome } \\
\text { original do } \\
\text { instrumento }\end{array}$} & \multicolumn{2}{|c|}{ Reprodutibilidade "teste-reteste" } & \multicolumn{2}{|c|}{ Validade } & \multirow[t]{2}{*}{ Período * } & \multirow[t]{2}{*}{ Capacidade $\star \star$} \\
\hline & & $\begin{array}{l}\text { Características } \\
\text { da amostra }\end{array}$ & $\begin{array}{l}\text { Métodos e } \\
\text { resultados }\end{array}$ & $\begin{array}{l}\text { Características } \\
\text { da amostra }\end{array}$ & $\begin{array}{c}\text { Critério de referência } \\
\text { e resultados }\end{array}$ & & \\
\hline Kemper et al. 28 & PAQ & & & $\begin{array}{c}\mathrm{N}=200(\mathrm{M} / \mathrm{F}) \\
\text { Holanda } \\
\text { Idade: } 13-16 \\
\text { anos }\end{array}$ & $\begin{array}{c}\text { Pedômetro, 4D } \\
r=0,20 \\
\text { Monitoramento da } \\
\text { frequência cardíaca, } \\
2 D \\
r=0,17\end{array}$ & $3 \cup \mathrm{M}$ & 2 \\
\hline Murphy et al. 53 & $\begin{array}{c}\text { Three Activity } \\
\text { Posteres }\end{array}$ & & & $\begin{array}{c}\mathrm{N}=92(\mathrm{M} / \mathrm{F}) \\
\text { Estados Unidos } \\
\text { Idade: } 10-18 \\
\text { anos }\end{array}$ & $\begin{array}{l}\mathrm{VO}_{2} \max \\
\text { rho }=0,21\end{array}$ & ST & 2 \\
\hline Crocker 39 & PAQ-C & $\begin{array}{c}\mathrm{N}=84(\mathrm{M} / \mathrm{F}) \\
\text { Canadá } \\
\text { Idade: 9-14 anos }\end{array}$ & $\begin{array}{l}\text { Teste-reteste, } 1 \mathrm{~S} \\
\qquad \mathrm{CCl}=0,78\end{array}$ & & & U7D & 2 \\
\hline Breener et al. 37 & $\begin{array}{c}\text { Youth Risk } \\
\text { Behavior Survey } \\
\text { Questionnaire } \\
\text { - } 1999\end{array}$ & 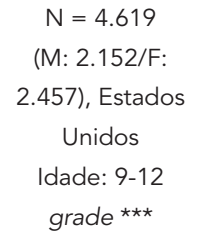 & $\begin{array}{c}\text { Teste-reteste, } \\
10-22 D \\
\text { kappa }=0,61\end{array}$ & & & U7D & 1 \\
\hline Zulling et al. 43 & $\begin{array}{l}\text { Middle School } \\
\text { Youth Risk } \\
\text { Behavior } \\
\text { Questionnaire } \\
\text { - } 2005\end{array}$ & $\begin{array}{c}\mathrm{N}=232 \\
\text { (M: 105/F: 127), } \\
\text { Estados Unidos } \\
\text { Idade: } 7-8 \\
\text { grade }\end{array}$ & $\begin{array}{c}\text { Teste-reteste, } 2 \mathrm{~S} \\
\text { kappa }=0,76\end{array}$ & & & U7D & 1 \\
\hline Brener et al. 36 & $\begin{array}{c}\text { Youth Risk } \\
\text { Behavior Survey } \\
\text { Questionnaire } \\
\text { - } 1992\end{array}$ & $\begin{array}{c}\mathrm{N}=1.679 \\
\text { (M: 774/F: 905), } \\
\text { Estados Unidos } \\
\text { Idade: } 7-12 \\
\text { grade }\end{array}$ & $\begin{array}{l}\text { Teste-reste, } 2 \mathrm{~S} \\
\text { kappa }=0,75\end{array}$ & & & U7D & 1 \\
\hline Gilmer et al. 41 & $\begin{array}{c}\text { Weekly Activity } \\
\text { Checklist }\end{array}$ & $\begin{array}{c}\mathrm{N}=103 \\
(\mathrm{M} / \mathrm{F}), \text { Estados } \\
\text { Unidos } \\
\text { Idade (média): } \\
\text { 12,3 anos; } \\
\mathrm{DP}=1,0\end{array}$ & $\begin{array}{c}\text { Teste-reteste, } 2 \mathrm{~S} \\
r=0,70\end{array}$ & & & US & 2 \\
\hline Brown \& Holland 38 & $\begin{array}{c}\text { Modified } \\
\text { Version Self- } \\
\text { Assessed } \\
\text { Physical Activity } \\
\text { Checklist }\end{array}$ & $\begin{array}{c}\mathrm{N}=103 \\
\text { (M: 52/F: 51), } \\
\text { Austrália } \\
\text { Idade (média): } \\
\text { 11,7 anos; } \\
\text { DP }=0,5\end{array}$ & $\begin{array}{l}\text { Teste-reteste, 5D } \\
\qquad \mathrm{CCl}=0,20\end{array}$ & & & UD & 1 \\
\hline Kriska et al. 42 & MAQ & $\begin{array}{c}\mathrm{N}=23 \\
\text { (M: 10/F: 13), } \\
\text { Estados Unidos } \\
\text { Idade: } 10-59 \\
\text { anos }\end{array}$ & $\begin{array}{c}\text { Teste-reteste, } \\
\qquad \begin{array}{c}1-3 S \\
\text { rho }=0,37\end{array}\end{array}$ & & & UA & 1 \\
\hline & $\begin{array}{l}\text { Entrevistas } \\
\text { estruturadas }\end{array}$ & & & & & & \\
\hline
\end{tabular}

(continua) 
Tabela 2 (continuação)

\begin{tabular}{|c|c|c|c|c|c|c|c|}
\hline \multirow[t]{2}{*}{ Referências } & \multirow{2}{*}{$\begin{array}{l}\text { Tipo e nome } \\
\text { original do } \\
\text { instrumento }\end{array}$} & \multicolumn{2}{|c|}{ Reprodutibilidade "teste-reteste" } & \multicolumn{2}{|c|}{ Validade } & \multirow[t]{2}{*}{ Período * } & \multirow[t]{2}{*}{ Capacidade ** } \\
\hline & & $\begin{array}{l}\text { Características } \\
\text { da amostra }\end{array}$ & $\begin{array}{l}\text { Métodos e } \\
\text { resultados }\end{array}$ & $\begin{array}{l}\text { Características } \\
\text { da amostra }\end{array}$ & $\begin{array}{l}\text { Critério de referência } \\
\text { e resultados }\end{array}$ & & \\
\hline Sallis et al. 24 & 7DPAR & $\begin{array}{c}\mathrm{N}=102 \\
\text { (M: 50/F: } 52 \text { ), } \\
\text { Estados Unidos } \\
\text { Idade (média): } \\
11,2 \text { anos; } \\
\text { DP }=0,6 \text { e } 16,4 ; \\
\text { DP }=0,7\end{array}$ & $\begin{array}{c}\text { Teste-reteste, 6D } \\
\qquad \mathrm{Cl}=0,77\end{array}$ & $\begin{array}{c}\mathrm{N}=102 \\
\text { (M: 50/F: } 52 \text { ), } \\
\text { Estados Unidos } \\
\text { Idade (média): } \\
11,2 \text { anos; } \\
\text { DP }=0,6 \text { e } 16,4 ; \\
\text { DP }=0,7\end{array}$ & $\begin{array}{l}\text { Monitoramento da } \\
\text { frequência cardíaca, } \\
\text { 1D } \\
r=0,49\end{array}$ & U7D & 1 \\
\hline Wallace et al. 60 & 7DPAR & & & $\begin{array}{l}\mathrm{N}=11(\mathrm{M}) \text {, } \\
\text { Estados Unidos } \\
\text { Idade (média): } \\
12,5 \text { anos; } \\
\mathrm{DP}=0,7\end{array}$ & $\begin{array}{l}\text { Observação direta do } \\
\text { comportamento, US } \\
\text { Não havia diferenças } \\
\text { significativas no gasto } \\
\text { energético total entre } \\
\text { os dois métodos }\end{array}$ & U7D & 1 \\
\hline Crocker et al. 16 & 7DPAR & & & $\begin{array}{c}\mathrm{N}=61 \\
\text { (M: 27/F: 34), } \\
\text { Canadá } \\
\text { Idade (média): } \\
\text { 11,2 anos; } \\
\text { DP =0,6 }\end{array}$ & $\begin{array}{c}\text { Acelerômetro - R3D, } \\
\begin{array}{c}\text { 7D } \\
r=-0,13 \\
\text { PAQ-C, U7D } \\
r=0,39\end{array}\end{array}$ & U7D & 1 \\
\hline $\begin{array}{l}\text { Simons-Morton et } \\
\text { al. } 32\end{array}$ & $\begin{array}{c}\text { Physical Activity } \\
\text { Record }\end{array}$ & & & $\begin{array}{c}\mathrm{N}=48 \\
\text { (M: 20/F: 28), } \\
\text { Estados Unidos } \\
\text { Idade: 7-11 anos }\end{array}$ & $\begin{array}{l}\text { Acelerômetro, 1D } \\
\qquad r=0,54 \\
\text { Monitoramento da } \\
\text { frequência cardíaca, } \\
\text { 1D } \\
r=0,62\end{array}$ & UD & 2 \\
\hline Larsson et al. 51 & PAI & & & $\begin{array}{c}N=32 \\
\text { (M: 17/F: 15), } \\
\text { Suécia } \\
\text { Idade: } 16-20 \\
\text { anos }\end{array}$ & $\begin{array}{c}\text { DLW, 14D } \\
\text { PAl subestima o } \\
\text { gasto energético total } \\
\text { nos mais ativos e } \\
\text { superestima nos menos } \\
\text { ativos; } 1,4 \pm 2,6 \mathrm{MJ} / \mathrm{d} \\
\text { (IC95\%: } 2,4-0,5 \mathrm{MJ} / \mathrm{d}) .\end{array}$ & 2US & 2 \\
\hline
\end{tabular}

(continua) 
Tabela 2 (continuação)

\begin{tabular}{|c|c|c|c|c|c|c|c|}
\hline \multirow[t]{2}{*}{ Referências } & \multirow{2}{*}{$\begin{array}{l}\text { Tipo e nome } \\
\text { original do } \\
\text { instrumento }\end{array}$} & \multicolumn{2}{|c|}{ Reprodutibilidade "teste-reteste" } & \multicolumn{2}{|c|}{ Validade } & \multirow[t]{2}{*}{ Período * } & \multirow[t]{2}{*}{ Capacidade ** } \\
\hline & & $\begin{array}{c}\text { Características } \\
\text { da amostra }\end{array}$ & $\begin{array}{l}\text { Métodos e } \\
\text { resultados }\end{array}$ & $\begin{array}{c}\text { Características } \\
\text { da amostra }\end{array}$ & $\begin{array}{c}\text { Critério de referência } \\
\text { e resultados }\end{array}$ & & \\
\hline \multirow[t]{6}{*}{ Cradock et al. 47} & 24h Recall & & & $N=43$ & Acelerômetro R3D, 3D & UD & 2 \\
\hline & Interview & & & (M: 26/F: 17), & & & \\
\hline & & & & Estados Unidos & $r=0,49$ & & \\
\hline & & & & Idade (média): & & & \\
\hline & & & & 13,8 anos; & & & \\
\hline & & & & $\mathrm{DP}=0,7$ & & & \\
\hline
\end{tabular}

APARQ: Adolescent Physical Activity Recall Questionnaire; CAAL: Child/Adolescent Activity Log; CAINM: Cuestionario de Actividad e Inactividad; B-A: diagrama de dispersão de Bland-Altman; CCl: coeficiente de correlação intraclasse; CDPAQ: Computer Delivered Physical Activity Questionnaire; CLASS: The Children's Leisure Activities Study Survey; DLW: doubly labelled water; DP; desvio-padrão; ES: especificidade; F: feminino;

GSPA: Godin-Shepard Physical Activity; IPAQ: International Physical Activity Questionnaire Short; M: masculino; MAQ: Modifiable Physical Activity Questionnaire; MARCA: Multimedia Activity Recall for Child and Adolescent; PACE+: Adolescent Physical Activity Measure; PDPAR: Previous Day Physical Activity Recall; PAI: Physical Activity Interview; PALQ Physical Activity and Lifestyle Questionnaire; PAQ: Physical Activity Questionnaire; PAQ-A: Physical Activity Questionnaire for Adolescent; PAQ-C: Physical Activity Questionnaire for Older Children; PS: Perspiration Score; QAPACE: Quantification de I'Activite Physique en Altitude Chez le Enfants; r: coeficiente de correlação de Pearson; rho: coeficiente de correlação de Spearman; SAPAC: Self-Administered Physical Activity Recall Checklist; SAPAQ: Self-Reported 7-Day Physical Activity Questionnaire; SAS: Specific Activity Score; SE: sensibilidade; SHAPES: School Health Action, Planning and Evaluation System; SS: Stairs Score; ST: Questionário de Atividade Física para Adolescentes; UA: Questionário de Atividade Física para Adolescentes; WHO HBSC: World Health Organization Questionnaire; YMCLS: Youth Media Campaign Longitudinal Survey; US: última semana; U7D: últimos 7 dias; 1D: 1 dia; $1 \mathrm{~h}: 1$ hora; 1S: 1 semana; 1-2S: 1-2 semanas; 1-3S: 1-3 semanas; 2D: 2 dias; 2S: 2 semanas; 3D: 3 dias; 3DAR: 3-Day Activity Record; 3DAR: 3-Day Aerobic Recall; 3DPAR: 3-Day Physical Activity Recall; 3DSR: 3-Day Sweat Recall; 7DPAR: 7-Day Physical Activity Recall; 4D: 4 dias; 4-5D: 4-5 dias; 5D: 5 dias; 6D: 6 dias; 6M: 6 meses; 6-8h: 6-8 horas; 6-10D: 6-10 dias; 7D: 7 dias; 8D: 8 dias; 9D: 9 dias; 10-22D: 10-22 dias; 11M: 11 meses; 12M: 12 meses; 1216D: 12-16 dias; 14D: 14 dias; 15D: 15 dias; 90D: 90 dias; < 24h: menos de 24 horas;

* Período de referência da medida do instrumento;

** Capacidade do instrumento de realizar estimativas do nível de atividade física dos adolescentes de acordo com as guias de recomendação de atividade física para adolescentes ( $1=\operatorname{sim}, 2=$ não);

*** Os autores não informaram a idade dos adolescentes (equivalência série de ensino e idade: $6=11-12$ anos; $7=12-13$ anos; $8=13-14$ anos; $9=14-15$ anos; $10=15-16$ anos; $11=16-17$ anos; $12=17-18$ anos).

reteste" - réplicas de aplicação. Nesse sentido, os resultados dos estudos analisados são referentes à reprodutibilidade "teste-reteste". O intervalo de tempo entre as aplicações variou, consideravelmente, de poucas horas 35,64 a 12 meses 76 . O período mais utilizado foi duas semanas ou mais (27/50) 17,19,20,24,36,37,41,42,43,61,67,68,69,70,74,76, $77,78,81,82$.

A maioria das medidas de reprodutibilidade "teste-reteste" (31/50) 17,19,23,24,26,27,29,34,38,39, $40,61,62,64,65,66,67,68,69,70,71,72,73,80,81,82$ foi estimada pelo coeficiente de correlação intraclasse (CCI). Também foram utilizados os coeficientes de correlação de Spearman (7/50) 20,42,76,77, Pearson (7/50) 18,22,35,41,63,74,79 e o índice kappa (5/50) $36,37,43,78,79$.

Os coeficientes de reprodutibilidade "testereteste" variaram de 0,20 38 a 0,98 26; a maioria (28/50; 56\%) 17,18,19,20,22,34,37,38,42,61,63,67,68,71,73,74, $76,77,78,79,80,82$ ficou abaixo de 0,70 . Vinte e dois instrumentos 23,24,26,27,29,35,36,39,40,41,43,61,62,64,35,66, $69,70,72,81$ alcançaram valores iguais ou superiores a 0,70 , e, dentre eles, nove $23,26,27,29,35,61,62,72,81$ apresentaram coeficientes de reprodutibilidade superiores a $0,90(9 / 22 ; 40,1 \%)$.

Seis 26,35,40,61,62,64 dos sete diários demonstraram coeficientes de reprodutibilidade "testereteste” superiores a 0,80. Dos 42 questionários analisados, apenas sete $23,24,27,29,70,72,81$ tinham níveis de reprodutibilidade superiores a 0,80. Encontrou-se uma única medida de reprodutibilidade para as entrevistas estruturadas $(\mathrm{CCI}=0,77) 24$.

Poucos estudos compararam os coeficientes de reprodutibilidade por sexo, idade e por intensidade das atividades físicas. Dentre os que compararam, a maioria demonstrou maior reprodutibilidade nas medidas de atividade física dos rapazes (9/15) 24,37,63,64,69,73,77,81, dos adolescentes mais velhos (15-19 anos; 6/7) 24,34,69,77,81 e nas atividades físicas vigorosas (12/16) 22,24,34,40, $63,64,69,73,74,77,79,80$. 


\section{Validade}

Cinquenta e oito estudos haviam analisado a validade de 71 instrumentos. Quarenta e seis 16,17 , $18,22,23,25,26,28,29,31,34,44,45,47,48,51,52,54,55,56,57,58,61,63$, $64,65,66,67,69,71,72,73,74,77,78,79,80,81,82$ eram referentes a estudos publicados entre 2000 e 2007; a maioria desses instrumentos (43/71) 16,18,19,20,21,22,24, $25,26,27,30,31,32,34,35,44,47,50,52,53,54,59,60,62,63,66,67,68,70$, 73,74,76,79 foi testada em adolescentes de poucos países da América do Norte, especialmente Estados Unidos $(\mathrm{n}=31$ ) 18,19,22,24,25,26,27,30,31,32,34,35, $44,47,52,53,54,59,60,63,66,67,68,70,73,76$, de ambos os sexos (61/71), mas não de diferentes faixas etárias (1014 e 15-19 anos, 41/71). O tamanho da amostra variou de 1160 a 2.026 sujeitos 78,82. A maioria tinha 50 sujeitos ou mais $(45 / 71) 16,17,18,20,21,22$, $24,27,28,29,30,31,34,44,46$, 50,52,53,54,55,58,62,64,65,66,67,69, $71,72,73,74,76,77,78,79,82$ e se tratava de amostras por conveniência $(42 / 71)$ 17,18,21,22,23,24,26,27,30,31,32,33 $34,44,46,48,49,50,51,52,53,54,57,58,59,60,62,64,66,67,68,69,70,71$, $72,73,77,79$ ou por participação voluntária (12/71) $16,19,25,28,45,56,63,65,80$.

Quinze instrumentos 16,21,23,26,27,28,29,30,31,32, $33,34,35,50$ haviam sido testados contra mais de um critério de referência - "padrão-ouro”, o que resultou em 90 medidas de validade. O coeficiente de correlação de Pearson foi a análise mais utilizada para estimar a validade dos instrumentos (58/90) 16,18,19,21,22,23,24,25,26,27,28,30,32,33,34,35,45,47,48 $50,52,54,61,62,63,65,66,68,70,74,80,81$, seguido pela correlação de Spearman (25/90) 9,17,20,29,31,44,53,55,56,57, $58,59,64,71,72,73,76,77,79,82$. O diagrama de dispersão de Bland-Altman 46,49,51, o índice kappa 69, o teste t para amostras independentes 46,51,60 e medidas de sensibilidade e especificidade 67 também foram utilizados.

Cinquenta e nove medidas de validade 16,18 , $19,21,22,23,24,25,26,27,28,29,30,31,32,33,34,35,44,45,47,48,50$, $51,54,55,56,57,58,59,60,61,63,64,65,66,67,70,71,72,73,79,80$ tiveram como "padrão-ouro" ao menos uma medida objetiva da atividade física. Dentre elas, a medida de atividade física por meio de acelerômetros foi o critério mais utilizado (40/90) 16, $18,19,21,22,23,24,25,26,27,31,32,33,34,35,44,47,48,50,54,55,59$, $61,63,65,66,67,70,71,72,73,79,80$. As medidas subjetivas foram relativamente pouco utilizadas como critério de referência $(20 / 90 ; 22 \%)$ 16,20,21, 24,29,33,34,50,68,69,74,77, sobretudo, como o critério principal (10/90) 20,21,24,68,69,74,77. Medidas de aptidão cardiorrespiratória também foram utilizadas como "padrão-ouro" para avaliar os níveis de validade dos instrumentos (testes de campo e de laboratório - medidas diretas e indiretas) (10/90) 17,21,27,52,53,62,76,78,81,82. A combinação de medidas de atividade física também foi adotada como "padrão-ouro": de medidas objetivas $23,26,28,30,32,35$, objetivas e subjetivas
$16,21,29,31,33,34,50$, e destas com de aptidão cardiorrespiratória 21,27 .

As medidas de validade variaram de -0,13 16 a 0,88 35; a maioria $(64 / 84 ; 76 \%) 16,17,18,19,20,21,22,24$, $25,26,27,28,29,30,31,33,34,44,47,50,52,53,54,55,57,58,59,62,63,64$, $65,66,68,69,71,72,73,74,76,77,79,80,82$ dos coeficientes de correlação foi igual ou inferior a 0,50; 16 21,23,25, $30,32,33,35,45,48,50,61,70,81$ tinham valores entre 0,51 0,69 ; quatro alcançaram valores iguais ou superiores a $0,7026,35,56$.

Parece haver uma relação entre a magnitude dos coeficientes de correlação para a validade e o tipo de critério de referência utilizado (Figura 2). Quase todas as correlações foram $\leq 0,50$ quando se compararam os instrumentos às medidas subjetivas da atividade física (17/20; 85\%) 16,20, $21,24,29,31,34,68,69,74,77$ ou de aptidão cardiorrespiratória (9/10; 90\%) 17,21,27,52,53,62,76,78,82. Nas medidas objetivas de atividade física, verificou-se uma menor frequência de correlações de magnitude muito baixa $(<0,30)$ e baixa $(\leq 0,50)(38 / 54$; $70,1 \%)$.

Ao considerar apenas os estudos que utilizaram medidas objetivas mais robustas (DWL, acelerômetros, monitores de frequência cardíaca) como "padrão-ouro", verificou-se que a maioria dos coeficientes de correlação - medida de validade - $(32 / 44 ; 72,7 \%)$ foi igual ou inferior a 0,50 (diários: 50\%; questionários: $80 \%$; entrevistas: $75 \%) ; 20,5 \%$ (9/44) entre 0,51-0,69 e 6,8\% (3/44) com valores iguais ou superiores a 0,70 .

Dentre as medidas de validade que foram comparadas segundo o sexo, idade e nível de intensidade das atividades físicas, a maioria evidenciou maiores níveis de validade nas medidas dos adolescentes do sexo masculino (15/22) 17,22, $28,29,33,44,45,55,63,66,69,70,77,81$, nos de maior idade (15-19 anos, 7/10) 24,32,34,65,69,77,81 e nas atividades físicas vigorosas (15/18) 18,22,24,34,54,58,59,64,67, $73,74,76,77,80$.

\section{Discussão}

Há uma grande diversidade de instrumentos de medida da atividade física do tipo self-report que foram testados (reprodutibilidade e validade) em adolescentes (10-19 anos), sobretudo questionários. Tais instrumentos mensuram de diferentes formas a atividade física, por diferentes períodos de tempo. Em geral, demonstraram maior reprodutibilidade "teste-reteste" do que validade. Maiores coeficientes de reprodutibilidade e validade foram relativamente mais frequentes nos diários do que nos questionários e entrevistas.

Trata-se da primeira revisão sistemática a analisar, de forma simultânea, diferentes carac- 


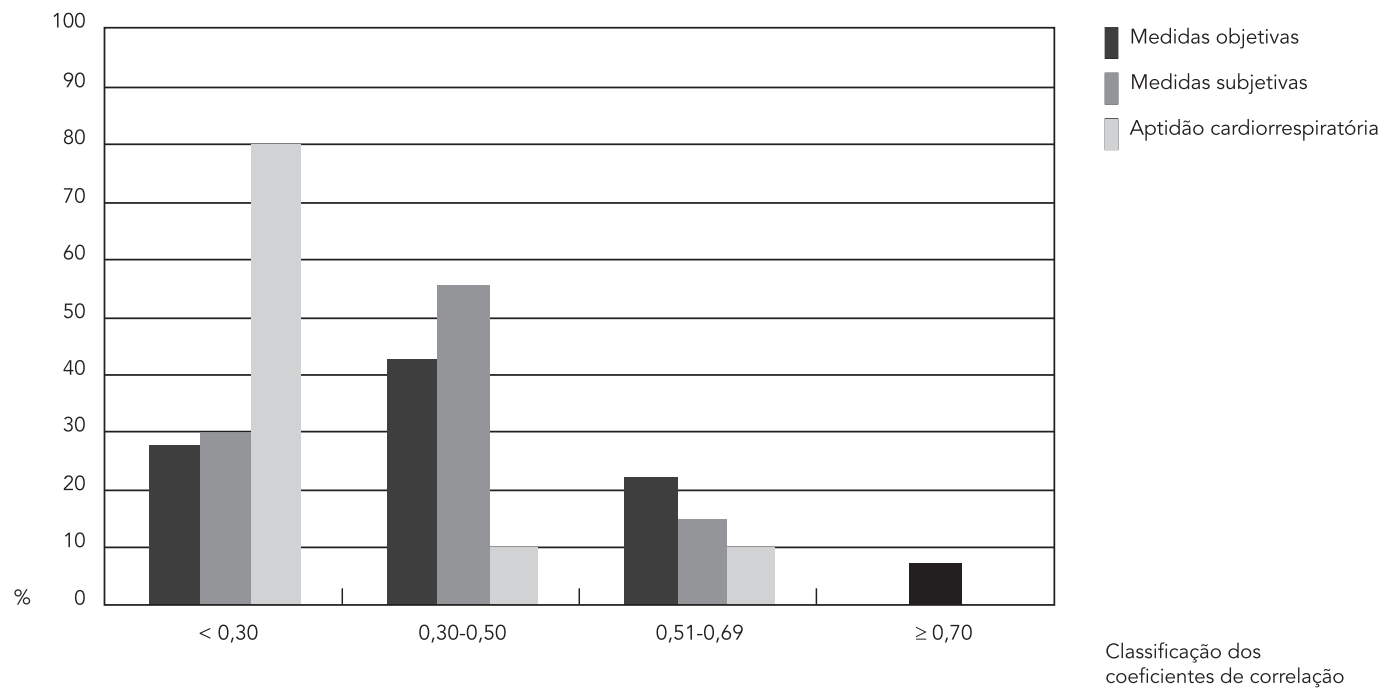

terísticas dos instrumentos self-report de medida da atividade física em adolescentes (além da reprodutibilidade e da validade) e os aspectos metodológicos desses estudos. Isto poderá ajudar pesquisadores e profissionais que pretendam mensurar a atividade física em adolescentes a responder uma questão comum: "Qual o instrumento devo utilizar?".

Este estudo apresenta algumas limitações que precisam ser levadas em conta. Uma das limitações foi não ter avaliado a qualidade metodológica dos estudos incluídos na revisão. Entretanto, destaca-se que os principais aspectos metodológicos que são avaliados (seleção da amostra, análise dos dados, qualidade das medidas utilizadas) fizeram parte da análise desta revisão. Além disso, as implicações das possíveis limitações dos estudos sobre os níveis de reprodutibilidade e validade dos instrumentos foram analisadas e discutidas.

Outra limitação foi não ter incluído os estudos que apresentavam os coeficientes de reprodutibilidade e/ou validade como parte dos métodos em estudos com outros objetivos. No entanto, acredita-se que os resultados não seriam diferentes, caso estes estudos tivessem sido incluídos. Deve-se considerar, também, a possibilidade de algum estudo que atendia aos critérios de inclusão não ter sido identificado pelos métodos de busca.
Mensurar a atividade física dos adolescentes, apesar de ser uma tarefa desafiadora, tem despertado o interesse de diversos pesquisadores em vários países, como indicado pelo número de instrumentos desenvolvidos e testados $(n=52)$, e de estudos publicados sobre as propriedades psicométricas desses instrumentos ( $\cong 65 \%$ entre 2000 e 2007).

Contudo, na maioria das vezes, semelhantes estudos parecem que mais contribuíram para adicionar novos instrumentos de medida da atividade física do que sistematicamente exploraram princípios de medida da atividade física em adolescentes. Por exemplo, apenas 11 instrumentos, dos 52 identificados nesta revisão, foram testados mais de uma vez. Além disso, a maioria dos estudos desenvolvidos ficou restrita a poucos países da América do Norte, não incluiu adolescentes de diferentes faixas etárias e utilizou sujeitos selecionados por conveniência ou participação voluntária.

Em relação às implicações da adoção desse tipo de seleção da amostra, não se pode descartar a hipótese de que os sujeitos sejam participantes mais motivados, podendo artificialmente inflar as medidas de reprodutibilidade e/ou de validade 83,84 . Outro aspecto que merece ser destacado é a possibilidade de ocorrência de "viés cultural" 84, caso esses instrumentos sejam aplicados diretamente (sem adaptação e testagem 
prévias) em amostras com características distintas das quais os mesmos foram desenvolvidos e avaliados. Dentre os 52 instrumentos identificados, apenas cinco 17,29,40,69,77 foram testados em adolescentes brasileiros.

A proliferação de instrumentos do tipo selfreport em adolescentes pode indicar três aspectos: a diversidade de interesses de pesquisa envolvendo a prática de atividade física; a relação "dose-resposta" da atividade física difere segundo o desfecho em estudo, exigindo medidas específicas; falta de consenso sobre quais instrumentos utilizar em determinadas situações.

A vantagem de se dispor de múltiplos instrumentos é a possibilidade de responder a diferentes objetivos em estudos com atividade física. Por sua vez, isso pode dificultar ou impossibilitar a comparação e interpretação de resultados dos estudos, pois há diferenças, às vezes acentuadas, na forma como os instrumentos operacionalizam e expressam o nível de atividade física dos adolescentes 8,12,83.

\section{Reprodutibilidade}

O procedimento de medidas repetidas foi utilizado para estimar todas as medidas de reprodutibilidade, conforme descrito em outras revisões $8,9,14,15$. Observou-se, porém, ampla variação no intervalo entre as réplicas de aplicação dos instrumentos, utilizando-se desde poucas horas até vários meses. Recomenda-se que o intervalo transcorrido entre as aplicações seja curto, de um a três dias, no máximo uma semana, e que haja sobreposição no período de recordação das atividades físicas praticadas nas duas aplicações $15,83,84$.

Desse modo, parece que vários estudos não examinaram a reprodutibilidade "teste-reteste" de forma adequada, pois utilizaram intervalos prolongados (mais de uma semana, $\mathrm{n}=27$ ) 17,19 , $20,24,36,37,41,42,43,61,67,68,69,70,74,76,77,78,81,82$ ou diferentes períodos de recordação nas duas aplicações (n = 23) 19,24,29,34,36,37,38,39,41,43,61,62,65,67,68,70, 74,77,78,79. Em função disso, muitos estudos estão tratando mudanças naturais na atividade física, ocorridas entre as réplicas de aplicação, como fonte de erro na estimativa da reprodutibilidade.

A maioria das medidas de reprodutibilidade “teste-reteste" foi estimada pelo CCI. Essa análise é considerada a mais apropriada para tal finalidade (dados contínuos e distribuição normal) 15,83,85. A correlação de Spearman foi utilizada para dados que não apresentaram uma distribuição normal e o índice kappa para dados categóricos (exemplo, suficientemente ativos $v s$. insuficientemente ativos).
Um dado preocupante em relação à análise estatística foi que $14 \% 18,22,35,41,63,74$ das medidas de reprodutibilidade foram estimadas pelo coeficiente de correlação de Pearson. Essa análise não deve ser utilizada para estimar reprodutibilidade. Primeiro, por se tratar de uma estatística bivariada, enquanto a situação em questão requer uma análise univariada 15,83,85; segundo, essa análise mede a magnitude da relação linear entre duas variáveis, sendo pouco sensível a pequenas variações que, normalmente, ocorrem no nível de atividade física entre as réplicas de aplicação do instrumento 15,83,85.

Houve uma grande variabilidade na medida de reprodutibilidade. Constatou-se que alguns instrumentos apresentaram apenas baixos níveis de reprodutibilidade $(\leq 0,50) \quad 19,20,38,42,67,71,74,78,79$ e outros níveis elevados (>0,95) 26,27,35,61,81. Vinte e dois instrumentos 23,24,26,27,29,35,36,39,40,41,43,61,62, $64,65,66,70,72,77,81$ demonstraram níveis de reprodutibilidade que podem ser considerados como satisfatórios $(\geq 0,70)$ 84,89 e 11 17,22,34,37,63,73,76,80,82 tiveram níveis moderados (0,60-0,69) 83,85.

Não há diferenças significantes na classificação dos níveis de reprodutibilidade entre a análise com e sem a inclusão dos coeficientes determinados por meio da correlação de Pearson $(<0$, 70: 28/50, 56\% vs. < 0,70: 23/43, 53,5\%). O mesmo foi observado para os coeficientes de maior magnitude (>0,90: 9/50, 18\% vs. 8/43, 18,6\%). Também não houve mudanças na amplitude dos coeficientes, permanecendo entre 0,20-0,98.

Baixos níveis de reprodutibilidade observados na maioria dos instrumentos pode ser uma indicação de que esses instrumentos apresentam problemas de clareza e objetividade. Outros fatores como a falta de padronização, mudanças na atividade física entre as réplicas de aplicações dos instrumentos também precisam ser considerados.

Maior reprodutibilidade "teste-reteste" nos diários pode ser atribuída à estrutura dos instrumentos (duração mensurada em blocos: 15min, 30min; atividades informadas em códigos/grupos) e ao fato de eles mensurarem as atividades físicas praticadas mais recentemente (exemplo, último dia, os últimos dois ou três dias) 4,8,12.

Poucos estudos $(<1 / 3)$ compararam os níveis de reprodutibilidade por sexo, idade e intensidade da atividade física. Dentre os que compararam, a maioria evidenciou maiores níveis de reprodutibilidade nas informações fornecidas pelos rapazes, pelos adolescentes mais velhos (15-19 anos) e nas atividades físicas de intensidade vigorosa. Possivelmente, isso se deve à maior participação dos rapazes em atividades físicas vigorosas, comparados com as moças, que tendem a ter maior participação em atividades 
físicas moderadas 4,8,12,75. Normalmente, as atividades vigorosas são estruturadas, planejadas e realizadas de forma repetitiva, sendo mais fáceis de serem recordadas 3 .

Em relação às possíveis influências da idade, acredita-se que o padrão de atividades físicas e as características psicológicas e cognitivas expliquem os resultados encontrados 3,8. Em geral, os adolescentes mais jovens apresentam maior envolvimento em atividades físicas esporádicas e de caráter intermitente, comparados aos seus pares mais velhos ${ }^{8,12}$. Estas atividades são mais difíceis de serem quantificadas e, portanto, recordadas 3 .

Níveis mais elevados de reprodutibilidade foram identificados nos instrumentos que mensuravam atividades físicas "recentes" comparados àqueles que mensuravam atividades habituais. Esses resultados reforçam indicações da literatura sobre a relação inversa entre precisão e tempo de recordação das atividades físicas 3,4,8,12,15,83,84 . A precisão com que as atividades físicas são recordadas diminui conforme aumenta o período de recordação.

\section{Validade}

A ausência de um "padrão-ouro", que seja adequado e consensual, contra o qual os instrumentos de medida da atividade física do tipo self-report - medidas subjetivas - sejam comparados ainda representa uma das principais lacunas nessa área 3,4,8,12,15,83,84. No presente estudo, foram identificados nove critérios de referência utilizados na validação: acelerômetros, pedômetros, monitores da frequência cardíaca, DLW, observação direta do comportamento, diários, questionários, entrevistas e medida da aptidão cardiorrespiratória.

Todos esses métodos apresentam algum tipo de limitação bem como mensuram de diferentes formas o nível de atividade física 4,5,8,12,15, produzindo diferentes resultados em termos de validade. Tal hipótese pode ser sustentada pelos achados desta revisão, que observou correlações de diferentes magnitudes para o mesmo instrumento (na mesma amostra), entre as medidas objetivas $23,26,28,30,32,35$, subjetivas 21,50 , objetivas e subjetivas $16,21,29,31,33,34,50$ e entre estas e as de aptidão cardiorrespiratória 21,27.

Por exemplo, os acelerômetros ("padrãoouro" mais utilizado) apresentam algumas particularidades e limitações: (1) diferentes equações têm sido utilizadas para determinar a intensidade das atividades físicas, produzindo resultados divergentes 44,86; (2) a validade desses equipamentos e as suas equações foram desenvolvidas em laboratório, podendo não ter o mesmo de- sempenho em condições de "vida real" 8,12; (3) mensuram com limitações algumas atividades e não podem ser utilizados em outras 8,12,86; (4) mensuram apenas atividades físicas "recentes" ou "atuais".

O coeficiente de correlação de Pearson e de Spearman foram as análises estatísticas mais utilizadas para determinar validade dos instrumentos. Essas análises são consideradas indicadores limitados de validade, uma vez que permitem avaliar, essencialmente, a magnitude da força da relação linear entre duas variáveis, mas não a concordância entre elas 83,87. Elas também não permitem avaliar a ocorrência de erro sistemático ou aleatório 87. Uma alternativa complementar é utilizar, quando possível (considerar pressupostos), medidas de sensibilidade/especificidade, o índice kappa e o método de Bland-Altman.

Uma grande variação foi observada nos coeficientes de validade, -0,13 a 0,88. Essa variabilidade é um indicativo de que muitos instrumentos e critérios de referência têm sido utilizados ${ }^{8}$. A maioria dos instrumentos apresentou coeficientes de correlação (medida de validade) de baixa magnitude $(\leq 0,50) 15,84$. Dentre os instrumentos analisados, apenas três apresentaram correlações $\geq 0,70$, todos eram diários: Previous Day Physical Activity Diary (PDPAR) 26,35 e Self-Report Activity Diary 56. Todos comparados com uma medida objetiva.

Os maiores coeficientes de correlação encontrados nos questionários variaram de 0,62 a 0,66. Cinco questionários alcançaram tais níveis de correlação: Computer Delivered Physical Actvity Questionnaire (CDPAQ, $\mathrm{r}=0,66$ ); Four by OneDay Recall Physical Activity Questionnaire ( $\mathrm{r}=$ 0,62); Physical Activity Questionnaire $(\mathrm{r}=0,63)$; Quantification de l'Activite Physique en Altitude Chez le Enfants (QAPACE, $\mathrm{r}=0,63$ ). Dentre as entrevistas analisadas, apenas uma apresentou correlação superior a 0,60 (Physical Activity Record, $\mathrm{r}=0,62)$. Medidas objetivas de atividade física serviram de critério de referência para avaliar todos os instrumentos, exceto o QAPACE (medida de $\mathrm{VO}_{2}$ máx por ergoespirometria).

Correlações de maior magnitude foram relativamente mais frequentes quando o critério de referência foi uma medida objetiva de atividade física, comparados às medidas subjetivas $\mathrm{e}$ de aptidão cardiorrespiratória. Esses resultados precisam ser interpretados com bastante cautela, dado que se trata, na maioria das vezes, de diferentes instrumentos, avaliados em amostras com diferentes características (por exemplo, faixa etária, número de sujeitos).

Particularmente em relação aos resultados dos estudos que utilizaram uma medida de aptidão cardiorrespiratória, os baixos coeficientes 
de correlação podem ser explicados pela grande carga genética desse indicador e por se tratar de uma medida de aptidão física e não de atividade física $4,8,9$.

Medidas objetivas de atividade física têm sido recomendadas para servir de critério de referência na validação das medidas subjetivas $4,5,8$. Pressupõe-se que esses métodos produzam estimativas mais robustas do nível de atividade física dos adolescentes, pois, não são influenciadas por viés de "memória" e de "estimação" 4,8,86.

Nesse sentido, realizou-se uma análise das evidências de validade dos instrumentos, levando em consideração apenas os estudos que tiveram como "padrão-ouro" uma medida objetiva "robusta" (acelerômetros, DLW, monitores de frequência cardíaca). Desse modo, 44 instrumentos foram analisados. A maioria $(72,7 \%)$ das correlações foi igual ou inferior a 0,50 (diários: 50\%; questionários: $80 \%$; entrevistas: $75 \%$ ). Considerando as correlações de magnitude moderada $(0,51-0,69)$ e moderada a elevada $(\geq 0,70), 18 \%$ $(n=9)$ e $6,8 \%(n=3)$, esses instrumentos apresentaram, respectivamente, tais coeficientes.

Estes resultados evidenciaram que mesmo utilizando apenas critérios de referências que podem ser considerados mais "robustos", a maioria dos instrumentos analisados nesta revisão apresentou baixos níveis de validade, sobretudo os questionários e as entrevistas. Melhores evidências de validade foram produzidas quando o "padrão-ouro" adotado foi uma medida objetiva de atividade física. Todavia, independentemente do "padrão-ouro", a maioria dos instrumentos analisados tem níveis de validade questionáveis.

Os baixos coeficientes de correlação que foram identificados na grande maioria dos instrumentos podem ser explicados de três formas: primeira, os instrumentos mensuram com limitações o nível de atividade física, pois dependem da capacidade dos adolescentes de recordar, interpretar e quantificar as atividades físicas praticadas - medidas subjetivas; segundo, os métodos de medida da atividade física apresentam limitações. Alguns instrumentos foram testados contra outras medidas subjetivas, cujos níveis de validade são desconhecidos. Isso tem sido um dos maiores problemas nessa área. Problemas de precisão no método de referência tendem a produzir subestimação da validade do instrumento que está sendo testado ou avaliado 88 . Terceiro, deve-se considerar que há, em várias ocasiões, uma "incompatibilidade" temporal (atividades "recentes" vs. típicas/habituais) e na operacionalização da medida de atividade física entre a medida do "padrão-ouro" e a do instrumento que está sendo testado. Os instrumentos mensuram de diferentes formas diferentes aspectos da atividade física.

Os diários apresentaram níveis mais elevados de validade do que os questionários e entrevistas, conforme descrito na literatura 4,8,9,12. Esses instrumentos foram os únicos que apresentaram correlações superiores a 0,70. Isso se deve, provavelmente, à forma como eles mensuram a atividade física (as mais recentes - minimiza "viés de memória"; as atividades são referidas em categorias/grupos; a duração é mensurada em blocos: 15min, 30min) e a utilização de uma medida objetiva como critério de referência (acelerômetros).

A maioria dos estudos relatou maior validade nas medidas fornecidas pelos rapazes, os adolescentes mais velhos (15-19 anos) e nas atividades físicas vigorosas, comparados às moças, aos adolescentes mais jovens (10-14 anos) e às atividades moderadas. Resultados similares foram descritos previamente 15. As possíveis explicações para esses achados são semelhantes às apresentadas para a medida de reprodutibilidade.

Foram identificados 52 instrumentos diferentes, a maioria deles questionários. Esses instrumentos mensuram de diferentes formas as dimensões e domínios da atividade física, por períodos de tempo que variam de um dia até os últimos 12 meses. Alguns instrumentos produzem uma medida geral de atividade física, outros fornecem informações detalhadas sobre as atividades físicas praticadas em um ou mais contextos.

Os instrumentos analisados no presente estudo, independentemente do tipo (diários, questionários, entrevistas), demonstraram maior reprodutibilidade "teste-reteste" do que validade. Em alguns instrumentos, os coeficientes de reprodutibilidade e validade foram elevados $(\geq 0,70)$. Entretanto, a maioria apresentou baixos níveis de reprodutibilidade, mas, sobretudo, de validade $(\leq 0,50)$, especialmente quando o "padrão-ouro" foi uma medida subjetiva. Poucas alterações foram verificadas nas evidências de validade ao considerar apenas os "padrões-ouro" mais robustos (acelerômetros, DLW, monitores de frequência cardíaca), sugerindo que a maioria dos instrumentos analisados mensura com limitações a atividade física dos adolescentes.

Em geral, maiores níveis de reprodutibilidade e de validade foram encontrados nos diários, se comparados aos questionários e entrevistas estruturadas. Contudo, há questionários que também alcançaram evidências aceitáveis de validade. Outros estudos são necessários para avaliar as evidências de reprodutibilidade e validade das entrevistas estruturadas. 
Os instrumentos que foram testados em adolescentes brasileiros demonstraram níveis moderados a elevados de reprodutibilidade "teste-reteste" e baixos níveis de validade. Novos estudos precisam ser desenvolvidos para testar outros instrumentos, particularmente utilizando uma medida objetiva de atividade física como "padrão-ouro".

\section{Resumo}

Realizou-se revisão sistemática de estudos de reprodutibilidade e validade de instrumentos de medida da atividade física do tipo self-report - medidas subjetivas, em adolescentes (10-19 anos). Buscas foram realizadas em bases de dados (MEDLINE, PsycInfo, SportsDiscus, Scopus, Web of Science, SciELO, Lilacs) $e$ nas referências dos artigos localizados. Sessenta e seis estudos atenderam aos critérios de inclusão. A maioria deles foi realizada em países da América do Norte, apenas cinco no Brasil. Identificaram-se 52 instrumentos diferentes: 42 questionários, 6 diários e 4 entrevistas. A reprodutibilidade "teste-reteste" variou de 0,20 a 0,98; a maioria (28/50) dos coeficientes apresentou valores $<$ 0,70 . Os coeficientes de validade apresentaram ampla variação $(-0,13$ a 0,88$)$, sendo a maioria deles $(64 / 84) \leq$ 0,50 . Apenas três instrumentos apresentaram correlações $\geq 0,70$. Diversos instrumentos foram testados em adolescentes, especialmente questionários. Em geral, tais instrumentos demonstraram melhor reprodutibilidade "teste-reteste" do que validade.

Atividade Motora; Adolescente; Reprodutibilidade dos Testes; Validade dos Testes; Métodos
Não há um instrumento que seja adequado a todos os estudos que envolvem medida de atividade física em adolescentes. Escolher o instrumento mais apropriado não depende somente dos níveis de reprodutibilidade e validade, mas também de outros fatores como as características da amostra, os objetivos do estudo, recursos disponíveis, os domínios e as dimensões da atividade física que serão mensurados e o período de referência da medida.

\section{Colaboradores}

J. C. Farias Júnior participou de todas as etapas do estudo, incluindo a busca e revisão dos estudos, análise e a redação do manuscrito. A. S. Lopes revisou e incluiu modificações à versão final do manuscrito. A. A. Florindo e P. C. Hallal participaram da estruturação dos métodos, análise dos resultados e da revisão crítica do manuscrito.

\section{Agradecimentos}

À Coordenação de Aperfeiçoamento de Pessoal de Nível Superior (CAPES), pela bolsa outorgada para a execução do estudo e aos autores dos artigos revisados, pelas informações fornecidas, em especial àqueles que enviaram as cópias eletrônicas dos artigos. 


\section{Referências}

1. Dumith SC. Physical activity in Brazil: a systematic review. Cad Saúde Pública 2009; 25 Suppl 3: S415-26.

2. Hallal PRC, Dumith SC, Bastos JP, Reichert FF, Siqueira FV, Azevedo MR. Evolução da pesquisa epidemiológica em atividade física no Brasil: revisão sistemática. Rev Saúde Pública 2007; 41:453-60.

3. Baranowski T. Validity and reliability of self-report measures of physical activity: an informationprocessing perspective. Res Q Exerc Sport 1988; 59:314-27.

4. Corder K, Ekelund U, Steele RM, Wareham NJ, Brage S. Assessment of physical activity in youth. J Appl Physiol 2008; 105:977-87.

5. Dollman J, Okely AD, Hardy L, Timperio A, Salmon J, Hills AP. A hitchhiker's guide to assessing young people's physical activity: deciding what method to use. J Sci Med Sport 2009; 12:518-25.

6. Bouchard C, Shephard RJ. Physical activity, fitness, and health: the model and key concepts. In: Bouchard C, Shephard RJ, Stephens T, editors. Physical activity, fitness and health: international proceedings and consensus statement. Toronto: Human Kinetics Publishers; 1994. p. 11-23.

7. Caspersen CJ, Poweell KE, Christenson GM. Physical activity, exercise, and physical fitness: definitions and distinctions for health-related research. Public Health Rep 1985; 100:126-31.

8. Sirard JR, Pate RR. Physical activity assessment in children and adolescents. Sports Med 2001; 31:439-54.

9. Kohl HW, Fulton JE, Caspersen CJ. Assessment of physical activity among children and adolescents: a review and synthesis. Prev Med 2000; 31:S54-76.

10. Reilly JJ, Penpraze V, Hislop J, Davies G, Grant S, Paton JY. Objective measurement of physical activity and sedentary behaviour: review with new data. Arch Dis Child 2008; 93:614-9.

11. Saris WHM. The assessment and evaluation of daily physical activity in children. A review. Acta Pediatr Scand Suppl 1985; 318:37-48.

12. Armstrong N, Welsman JR. The physical activity patterns of European youth with reference to methods of assessment. Sports Med 2006; 36: 1067-86.

13. Pate RR. Physical activity assessment in children an adolescents. Crit Rev Food Sci Nutr 1993; 33:321-6.

14. Sallis JF. Self-report measures of children's physical activity. J Sch Health 1991; 61:215-9.

15. Sallis JF, Saelens BE. Assessment of physical activity by self-report: status, limitations, and future directions. Res Q Exerc Sport 2000; 71(2 Suppl): S1-14.

16. Crocker PRE, Holowachuk DR, Kowalski KC. Feasibility of using the tritrac motion sensor over a 7-day trial with older children. Pediatr Exerc Sci 2001; 13:70-81.

17. Florindo AA, Romero A, Peres SV, Silva MV, Slater B. Desenvolvimento e validação de um questionário de avaliação da atividade física para adolescentes. Rev Saúde Pública 2006; 40:802-9.
18. Gao S, Harnack L, Schmitz K, Fulton J, Lytle L, Coevering PV. Reliability and validity of a brief tool to measure children's physical activity. J Phys Act Health 2006; 3:415-22.

19. Janz K, Witt J, Mahoney LT. The stability of children's physical activity as measured by accelerometry and self-report. Med Sci Sports Exerc 1995; 27:1326-32.

20. Koo MM, Rohan TE. Comparison of four habitual physical activity questionnaire in girls aged 7-15 yr. Med Sci Sports Exerc 1999; 31:421-7.

21. Kowalski KC, Crocker PRE, Faulkner RA. Validation of the physical activity questionnaire for older children. Pediatr Exerc Sci 1997; 9:174-86.

22. McMurray RG, Ring KB, Treuth MS, Welk GJ, Pate $\mathrm{RR}$, Schmitz KH, et al. Comparison of two approaches to structured physical activity surveys for adolescents. Med Sci Sports Exerc 2004; 36: 2135-43.

23. Ridley K, Dollman J, Olds T. Development and validation of a computer delivered physical activity questionnaire (CDPAQ) for children. Pediatr Exerc Sci $2001 ; 13: 35-46$.

24. Sallis JF, Buono MJ, Roby JJ, Micale FG, Nelson JA. Seven-day recall and other physical activity self-reports in children and adolescents. Med Sci Sports Exerc 1993; 25:99-108.

25. Welky GJ, Dzewaltowski DA, Hill JL. Comparison of the computerized ACTIVITYGRAM instrument and the previous day physical activity recall for assessing physical activity in children. Res Q Exerc Sport 2004; 75:370-80.

26. Allor KM, Pivarnik JM. Stability and convergent validity of three physical activity assessments. Med Sci Sports Exerc 2001; 33:671-6.

27. Garcia AW, George TR, Coviak C, Antonakos C, Pender NJ. Development of the child/adolescent actvity log: a comprehesive and feasible measure of leisure-time physical activity. Int J Behav Med 1997; 4:323-38.

28. Kemper HCG, Bakker I, Twisk JWR, van Mechelen W. Validation of a physical activity questionnaire to measure the effect of mechanical strain on bone mass. Bone 2002; 30:799-804.

29. Nahas MVN, Barros MVG, Florindo AA, Farias Júnior JC, Hallal PC, Konrad L, et al. Reprodutibilidade e validade do questionário saúde na boa para avaliar atividade física e hábitos alimentares em escolares do ensino médio. Rev Bras Ativ Fís Saúde 2007; 12:12-20.

30. Sallis JF, Strikmiller PK, Harsha DW, Feldman HA, Ehlinger S, Stone EJ. Validation of interviewer and self-administered physical activity checklists for fifht grade students. Med Sci Sports Exerc 1996; 28:840-51.

31. Scerpella TA, Tuladhar P, Kanaley JA. Validitation of the Godin-Shephard Questionnaire in prepubertal girls. Med Sci Sports Exerc 2002; 34:845-50.

32. Simons-Morton BG, Taylor WC, Huang IW. Validity of the physical activity interview and caltrac with preadolescent children. Res Q Exerc Sport 1994; 65:84-8 
33. Verheul AM, Prins AN, Kemper HGC, Kardinaal AFM, Erp-Baart MJV. Validation of a weight-bearing physical activity questionnaire in a study of bone density in girls and women. Pediatr Exerc Sci 1998; 10:38-47.

34. Welky GJ, Wickel E, Peterson M, Heitzler CD, Fulton JE, Potter LD. Reliability and validity of questions on the youth media campaign longitudinal survey. Med Sci Sports Exerc 2007; 39:612-22.

35. Weston AT, Petosa R, Pate RR. Validation of an instrument for measurement of physical activity in youth. Med Sci Sports Exerc 1997; 29:138-43.

36. Brener ND, Collins JL, Kann L, Warren CW, Williams BI. Reliability of the youth behavior survey questionnaire. Am J Epidemiol 1995; 141:575-80.

37. Brener ND, Kann L, McManus T, Kinchen SA, Sundberg EC, Ross JG. Reliability of the 1999 Youth Risk Behavior Survey Questionnaire. J Adolesc Health 2002; 31:336-42.

38. Brown TD, Holland BV. Test-retest reliability of the self-assessed physical activity checklist. Percept Mot Skills 2004; 99:1099-102.

39. Crocker PRE, Bailey DA, Faulkner RA, Kowalski $\mathrm{KC}$, McGrath R. Measuring general levels of physical activity: preliminary evidence for the physical activity questionnaire for older children. Med Sci Sports Exerc 1997; 29:1344-9.

40. Farias Júnior JC, Pires MC, Lopes AS. Reprodutibilidade de um questionário para o levantamento de informações sobre comportamentos relacionados à saúde em adolescentes. Rev Bras Ciênc Mov 2002; 10:43-8.

41. Gilmer MJ, Speck BJ, Bradley C, Harrel JS, Belyea M. The youth health survey: reliability and validity on an instrument for assessing cardiovascular health habits in adolescentes. J Sch Health 1996; 66:106-11.

42. Kriska AM, Knowler WC, LaPorte RE, Drash AL, Wing KR, Blair SN, et al. Development of questionnaire to examine relationship of physical activity and diabetes in Pima Indians. Diabetes Care 1990; 13:401-11.

43. Zulling KJ, Pun S, Patton JM, Ubbes VA. Reliability of the 2005 middle school youth risk behavior survey. J Adolesc Health 2006; 39:856-69.

44. Anderson CB, Hagströmer M, Yngve A. Validation of the PDPAR as an adolescent diary: effects of accelerometer cut points. Med Sci Sports Exerc 2005; 37:1224-30.

45. Arvidsson D, Slinde F, Hulthén L. Physical activity questionnaire for adolescents validated against doubly labelled water. Eur J Clin Nutr 2005; 59: 376-83.

46. Bratteby L-E, Sandhagen B, Fan H, Samuelson G. A 7-day activity diary for assessment of daily energy expenditure validated by the doubly labelled water method in adolescents. Eur J Clin Nutr 1997; 51:585-91.

47. Cradock AL, Wiecha JL, Peterson KE, Sobol AM, Colditz GA, Gortmaker SL. Youth recall and tritrac accelerometer estimates of physical activity levels. Med Sci Sports Exerc 2004; 36:525-32.
48. Ekelund U, Neovius M, Linné Y, Rössner S. The criterion validity of a last 7-day physical activity questionnaire (SAPAQ) for use in adolescents with a wide variation in body fat: the Stockholm Weight Development Study. Int J Obes (Lond) 2006; 30:1019-21.

49. Ekelund U, Yngvel A, Sjostrom M. Total daily energy expenditure physical activity in adolescents by two different methods. Scand J Med Sci Sports 1999; 9:257-64.

50. Kowalski KC, Crocker PRE, Kowalski NP. Convergent validity of the physical activity questionnaires for adolescents. Pediatr Exerc Sci 1997; 9:342-52.

51. Larsson C, Westerterp KR, Johansson GJ. Validity of reported energy expenditure and energy and protein intakes in swedish adolescent vegans and omnivores. Am J Clin Nutr 2002; 75:268-74.

52. Moore JB, Hanes Jr. JC, Barbeau P, Gutin B, Treviño RP, Yin Z. Validation of the Physical Activity Questionnaire for Older Children in children of different races. Pediatr Exerc Sci 2007; 19:6-19.

53. Murphy JK, Alpert BS, Dupaul LM, Willey ES, Walker SS, Nanney GC. The validity of children's selfreport of physical activity: a preliminary study. J Hum Hypertens 1990; 4:130-2.

54. Pate RR, Ross R, Dowda M, Trost SG, Sirard J. Validation of a 3-day physical activity recall instrument in female youth. Pediatr Exerc Sci 2003; 15:257-65.

55. Ridder CM, Kemper HCG, Bertensy MJM, van Gameren ACL, Ras E, Voogd J. Concurrent validity of a weight-bearing activity questionnaire in prepubertal and pubertal girls and boys. Ann Hum Biol 2002; 29:237-46.

56. Rodriguez G, Béghin L, Michaud L, Moreno LA, Turck D, Gottrand F. Comparison of the TriTracR3D accelerometer and self-report activity diary with heart-rate monitoring for the assessment of energy expenditure in children. Br J Nutr 2002; 87:623-31.

57. Slinde F, Arvidsson D, Sjoeberg A, RossanderHulthen L. Minnesota leisure time activity questionnaire and doubly labeled water in adolescents. Med Sci Sports Exerc 2003; 35:1923-8.

58. Trost SG, Marshall AL, Miller R, Hurley JT, Hunt JA. Validation of a $24-\mathrm{h}$ physical activity recall in indigenous and non-indigenous australian adolescents. J Sci Med Sport 2007; 10:428-35.

59. Trost SG, Ward DS, McGraw B, Pate RR. Validity of the previous day physical activity recall (PDPAR) in fifth-grade children. Pediatr Exerc Sci 1999; 11:341-5.

60. Wallace JP, Mckenzie TL, Nader PR. Observed vs. recalled exercise behavior: a validation of a seven day exercise recall for boys 11 to 13 years old. Res Q Exerc Sport 1985; 56:161-5.

61. Argiropoulou EC, Michaloupoulou M, Aggeloussis $\mathrm{N}$, Avgerinos A. Validity and reliability of physical activity measures in greek high school age children. J Sport Sci Med 2004; 3:147-59.

62. Bouchard C, Tremblay A, Leblanc C, Lortie G, Sauard R, Theriault G. A method to assess energy expenditure in children and adults. Am J Clin Nutr 1983; 37:461-7. 
63. Eisenmann JC, Milburn N, Jacobsen L, Moore SJ. Reliability and convergent validity of the Godin leisure-time exercise questionnaire in rural 5thgrade school-children. Journal of Human Movement Studies 2002; 43:135-49.

64. Lee K, Trost SG. Validity and reliability of the 3-day physical activity recall. Res Q Exerc Sport 2005; 76:101-6.

65. Mota J, Santos P, Guerra S, Ribeiro JC, Duarte JA, Sallis JF. Validation of a physical activity self-report questionnaire in a portuguese pediatric population. Pediatr Exerc Sci 2002; 14:259-76.

66. Prochaska JJ, Sallis JF, Long B. A physical activity screening measure for use with adolescents in primary care. Arch Pediatr Adolesc Med 2001; 155:554-9

67. Troped PJ, Wiecha JL, Fragala MS, Matthews CE, Finkelstein DM, Kim J, et al. Reliability and validity of YRBS physical activity items among middle school students. Med Sci Sports Exerc 2007; 39: 416-25.

68. Ching PLYH, Dietz WH. Reliability and validity of activity measures in preadolescents girls. Pediatr Exerc Sci 1995; 7:389-99.

69. Guedes DP, Lopes CC, Guedes JERP, Stanganelli LC. Reprodutilibidade e validade do questionário Baecke para avaliação da atividade física habitual em adolescentes. Rev Port Ciênc Desporto 2006; 6:265-74.

70. McMurray RG, Harrell JS, Bradley CB, Webb JP, Goodman EM. Comparison of a computerized physical activity recall with a triaxial motion sensor in middle-school youth. Med Sci Sports Exerc 1998; 30:1238-45.

71. Telford A, Salmon J, Jolley D, Crawford D. Reliability and validity of physical activity questionnaires for children: the children's leisure activities study survey (CLASS). Pediatr Exerc Sci 2004;16:64-78.

72. Ridley K, Olds TS, Hill A. The Multimedia Activity Recall for Children and Adolescents (MARCA): development and evaluation. Int J Behav Nutr Phys Act 2006; 3:10.

73. Treuth MS, Hou N, Young DR, Maynard M. Validity and reliability of the fels physical activity questionnaire for children. Med Sci Sports Exerc 2005; 37:488-95.

74. Hernández B, Gortmaker SL, Laird NM, Colditz GA. Parra-Cabrera S, Peterson KE. Validez y reproducibilidad de um questionario de actividad e inactividad física para escolares de la ciudad de México. Salud Pública Méx 2000; 42:315-23.

75. Biddle S, Sallis JF, Cavill NA. Young and active? Young people and health enhancing physical activity: evidence and implication. London: Health Education Authority; 1998.
76. Aaron DJ, Kriska AM, Dearwater SR, Cauley JA, Metz KF, LaPorte RE. Reproducibility and validity of an epedemiologic questionnaire to assess part year physical activity in adolescents. Am J Epidemiol 1995; 142:191-201.

77. Guedes DP, Lopes CC, Guedes JERP. Reprodutibilidade e validade do questionário internacional de atividade física em adolescentes. Rev Bras Med Esporte $2005 ; 11: 151-8$.

78. Booth ML, Okely AD, Chey T, Bauman A. The reliability and validity of the physical activity questions in the WHO health behaviour in schoolchildren (HSBC) survey: a population study. Br J Sports Med 2001; 35:263-7.

79. Wong SL, Leatherdale ST, Manske SR. Reliability and validity of a school-based physical activity questionnaire. Med Sci Sports Exerc 2006; 38: 1593-600.

80. Philippaerts RM, Matton L, Wijndaele K, Balduck AL, De Bourdeaudhuij I, Lefevre J. Validity of a physical activity computer questionnaire in 12- to 18-year-old boys and girls. Int J Sports Med 2006; 27:131-6.

81. Barbosa N, Sanchez C, Vera J, Perez W, Thalabard JC, Rieu M. A physical activity questionnaire: reproducibility and validity. Journal of Sports Science and Medicine 2007; 6:505-18.

82. Booth ML, Okely AD, Chey T, Bauman A. The reliability and validity of the adolescent physical activity recall questionnaire. Med Sci Sports Exerc 2002; 34:1986-95.

83. Patterson P. Reliability, validity, and methodological response to the assessment of physical activity via self-report. Res Q Exerc Sport 2000; 71 (2 Suppl):S15-20.

84. Shephard R. Limits to the measurement of habitual physical activity by questionnaires. Br J Sports Med 2004; 37:197-206.

85. Baumgartner TA, Chung H. Confidence limits for intraclass reliability coeficients. Measurement in Physical Education and Exercise Science 2001; 5:179-88.

86. Reilly J, Penpraze V, Hislop J, Davies G, Grant S, Paton J. Objective measurement of physical activity and sedentary behaviour: review with new data. Arch Dis Child. 2008; 93:614-9.

87. Bland JM, Altman G. Statistical methods for assessing agreement between two methods of clinical measurement. Lancet 1986; 327:307-10.

88. Monteiro CA, Florindo AA, Claro RM, Moura EC. Validade de indicadores de atividade física e sedentarismo obtidos por inquérito telefônico. Rev Saúde Pública 2008; 42:575-81.

Recebido em 14/Jan/2010

Versão final reapresentada em 30/Mai/2010

Aprovado em 11/Jun/2010 